\title{
ISOTOPY GROUPS
}

\author{
LAWRENCE L. LARMORE ${ }^{1}$
}

\begin{abstract}
For any mapping $f: V \rightarrow M$ (not necessarily an embedding), where $V$ and $M$ are differentiable manifolds without boundary of dimensions $k$ and $n$, respectively, $V$ compact, let $[V \subset M]_{f}=$ $\pi_{1}\left(M^{V}, E, f\right)$, i.e., the set of isotopy classes of embeddings with a specific homotopy to $f(E=$ space of embeddings). The purpose of this paper is to enumerate $[V \subset M]_{f}$. For example, if $k>3, n=2 k$, and $M$ is simply connected, [ $\left.S^{k} \subset M\right]_{f}$ corresponds to $\pi_{2} M$ or $\pi_{2} M \otimes Z_{2}$, depending on whether $k$ is odd or even. In the metastable range, i.e., $3(k+1)>2 n$, a natural Abelian affine structure on $[V \subset M]_{f}$ is defined: if, further, $f$ is an embedding $[V \subset M]_{f}$ is then an Abelian group. The set of isotopy classes of embeddings homotopic to $f$ is the set of orbits of the obvious left action of $\pi_{1}\left(M^{V}, f\right)$ on $[V \subset M]_{f}$.

A spectral sequence is constructed converging to a theory $H^{*}(f)$. If $3(k+1)<2 n, H^{0}(f) \cong[V \subset M]_{f}$ provided the latter is nonempty. A single obstruction $\Gamma(f) \in H^{1}(f)$ is also defined, which must be zero if $f$ is homotopic to an embedding; this condition is also sufficient if $3(k+1)<$ $2 n$. The $E_{2}$ terms are cohomology groups of the reduced deleted product of $V$ with coefficients in sheaves which are not even locally trivial. $\left[S^{k} \subset M\right]_{f}$ is specifically computed in terms of generators and relations if $n=2 k$, $k \geqslant 3$ (Theorem 6.0.2).
\end{abstract}

1. Introduction. In this paper, in some respects a sequel to [7], we attack the general problem of classifying, up to isotopy, embeddings of a compact $k$-manifold $V$ in an $n$-manifold $M$ in the metastable range, i.e., where $3(k+1)<2 n$.

Differentiable shall mean infinitely differentiable, manifold shall mean differentiable manifold without boundary (either compact or open), with a countable base; embedding shall mean differentiable embedding, and isotopy shall mean homotopy of embeddings.

Let $[V \subset M]$ denote the set of isotopy classes of embeddings of $V$ in $M$. Computation of $[V \subset M]$ is the ultimate goal. Unfortunately, $[V \subset M]$ has no convenient algebraic structure. Thus we introduce a new object, $[V \subset$ $M]_{f}$, where $f: V \rightarrow M$ is a specific map. A homotopy $f_{t}: V \rightarrow M, 0 \leqslant t \leqslant 1$, is

Received by the editors September 28, 1973 and, in revised form, September 7, 1976.

AMS (MOS) subject classifications (1970). Primary 57D40, 55G35; Secondary 55H25.

Key words and phrases. Isotopy, obstructions to embedding, twisted cohomology.

'The author wishes to thank Robert D. Rigdon, who gave him invaluable help in reorganizing the pair fibration concepts.

(1) American Mathematical Society 1978 
called an embedding-homotopy (or $e$-homotopy) of $f$ if $f_{0}=f$ and $f_{1}$ is an embedding. If $f_{u, t}: V \rightarrow M$, for $0 \leqslant t, u<1$ is a two-parameter homotopy such that, for all $u, f_{u, 0}=f$ and $f_{u, 1}$ is an embedding, we say that the $e$-homotopies $\left\{f_{0, t}\right\}$ and $\left\{f_{1, t}\right\}$ are isotopic. $[V \subset M]_{f}$ is then defined to be the set of isotopy classes of $e$-homotopies of $f$. If $\left\{f_{t}\right\}$ is an $e$-homotopy of $f$, let $\left[f_{t}\right] \in[V \subset M]_{f}$ be its isotopy class. It shall be shown that $[V \subset M]_{f}$ is an Abelian affine group in the metastable range, and an actual Abelian group if $f$ is an embedding.

If $2 k-n<0$, the group $\left[S^{k} \subset M^{n}\right]_{f}$ is, in this paper, expressed in terms of generators and relations, involving only $\pi_{1} M$ if $n=2 k+1$, and involving both $\pi_{1} M$ and $\pi_{2} M$ if $n=2 k$. For $n>2 k+1,\left[V^{k} \subset M^{n}\right]_{f}=0$.

Generally (although not shown in this paper) the affine structure of $\left[V^{k} \subset M^{n}\right]_{f}$ in the metastable range depends only on the homotopy of $M$ through dimension $2 k-n+2$, as well as on $V$.

The case $n=2 k+1$ was done in [7], but the result was incorrectly stated. See Theorem 6.0.1 for the correct version. (Another error in [7], an invalid proof of Theorems 3.3.1 and 3.3.2, is corrected here in §8. The error was pointed out by the referee of this paper.) The case $n=2 k$ is computed for the first time in this paper, and involves evaluation of one nonzero differential and one nontrivial extension in a spectral sequence whose $E_{2}$ terms are cohomology groups of the reduced deleted product of $S^{k}$ with coefficients in sheaves which do not, in general, have a local product structure. See Theorem 6.0.2 for the general result. Some specific cases are as follows (where $f$ is any embedding):

THEOREM 1.0.1. If $M=M^{2 k}$ is simply connected, $k \geqslant 3$, then:

$$
\left[S^{k} \subset M\right]_{f} \cong \begin{cases}\pi_{2} M \otimes Z_{2} & \text { if } k \text { is even, } \\ \pi_{2} M & \text { if } k \text { is odd. }\end{cases}
$$

THEOREM 1.0.2. If $k \geqslant 3$ :

$$
\left[S^{k} \subset P^{2} \times R^{2 k-2}\right]_{f} \cong \begin{cases}Z_{2}+Z_{2} & \text { if } k \text { is even, } \\ Z+Z+Z_{2} & \text { if } k \text { is odd. }\end{cases}
$$

THEOREM 1.0.3. If $k>3$ :

$$
\left[S^{k} \subset P^{2 k}\right]_{f} \cong \begin{cases}0 & \text { if } k \equiv 0 \bmod 4 \\ Z_{4} & \text { if } k \equiv 1 \bmod 4 \\ Z_{2} & \text { if } k \equiv 2 \bmod 4 \\ Z_{2}+Z_{2} & \text { if } k \equiv 3 \bmod 4\end{cases}
$$

Let $\pi=\pi_{1}\left(M^{V}, f\right)$. We may define a left action:

$$
\mu: \pi \times[V \subset M]_{f} \rightarrow[V \subset M]_{f}
$$


as follows: if $\left\{f_{t}\right\}$ is an $e$-homotopy of $f$ and if $\left\{f_{t}^{\prime}\right\}$ is a self-homotopy of $f$ representing $a \in \pi$, then $\mu\left(a,\left[f_{t}\right]\right)=\left[f_{t}^{\prime \prime}\right]$ where $f_{t}^{\prime \prime}=f_{2 t}$ or $f_{2 t-1}^{\prime}$, depending on the value of $t$. The action $\mu$ respects the affine structure of $[V \subset M]_{f}$, i.e., for each $a \in \pi, \mu(a$,$) is an affine automorphism (cf. Theorem 2.7.1).$

Now let $\phi:[V \subset M]_{f} \rightarrow[V \subset M]$ be the forgetful function, i.e., if $\left\{f_{t}\right\}$ is an $e$-homotopy of $f, \phi\left[f_{t}\right]=\left[f_{1}\right]$, the isotopy class containing $f_{1}$. Trivially, the reader can convince himself of the following:

(i) Every element of $[V \subset M]$ lies in the image of $\phi$ for some choice of $f$.

(ii) If $x, y \in[V \subset M]_{f}$, then $\phi x=\phi y$ if and only if $\mu(a, x)=y$ for some $a \in \pi$.

Classification, up to isotopy, of embeddings of $V$ in $M$ in the stable range may thus be reduced to the following two problems:

I. Compute the affine structure of $[V \subset M]_{f}$, for each $f: V \rightarrow M$.

II. Determine the action $\mu$ of $\pi_{1}\left(M^{V}, f\right)$ on $[V \subset M]_{f}$.

It is to the first of these problems that this paper is addressed.

In $\$ 2$, we use Haefliger's results [4] to define the affine structure on $[V \subset M]_{f}$. An alternative, equivalent definition of the affine structure is given in \$4; this definition is much more geometric and easier to comprehend, but it is the Haefliger definition that yields to computation, by homotopy methods. In $\$ 3$, a spectral sequence is defined, in $\$ 5$, the sheaves we need are computed, and in $\$ 6$ the spectral sequence is fully worked in the special cases.

Using somewhat different methods, Dax [2] and Salomonsen [12] have obtained results on the same and similar problems.

2. The affine structure of $[V \subset M]$.

2.1. Pair fibrations and weak pair fibrations. Following [11], we say that a map $p:\left(E, E_{0}\right) \rightarrow\left(X, X_{0}\right)$ is a pair fibration if for any given map $h:\left(Y, Y_{0}\right) \rightarrow$ $\left(E, E_{0}\right)$ and every homotopy $f_{t}:\left(Y, Y_{0}\right) \rightarrow\left(X, X_{0}\right), 0<t<1$, such that $f_{0}=p h$, there exists a homotopy $h_{t}:\left(Y, Y_{0}\right) \rightarrow\left(E, E_{0}\right)$ such that $h_{0}=h$ and $p h_{t}=f_{t}$ for all $t$.

A section of a pair fibration is a map $g:\left(X, X_{0}\right) \rightarrow\left(E, E_{0}\right)$ such that $p g=1$ (the identity). A lifting of $f:\left(Y, Y_{0}\right) \rightarrow\left(X, X_{0}\right)$ to $p$ is a map $h:\left(Y, Y_{0}\right) \rightarrow$ $\left(E, E_{0}\right)$ such that $p h=f$. Sections and liftings are said to be homotopic if they are homotopic as sections or liftings (i.e., fiber homotopic).

All the theorems in $\$ \S 2$ and 3 which deal with sections of pair fibrations are equivalent to corresponding theorems which deal with liftings, since a lifting of $f$ to $p$ corresponds to a section of $f^{-1} p$ (where $f^{-1}$ is the usual pullback construction).

If $\left(X, X_{0}\right)$ is a topological pair, we say that $\left(A, A_{0}\right)$ is a sub-pair of $\left(X, X_{0}\right)$ if $A \subset X$ and $A_{0}=A \cap X_{0}$. Then $\left(\left(X, X_{0}\right),\left(A, A_{0}\right)\right)$ is a pair of pairs. If $X$ is a C.W. complex, and $X_{0}$ and $A$ are subcomplexes, it is a C.W. pair of pairs.

For the following definitions, let (as needed) $f:\left(\left(Y, Y_{0}\right),\left(B, B_{0}\right)\right) \rightarrow$ 
$\left(\left(X, X_{0}\right),\left(A, A_{0}\right)\right)$ be a map of pairs of pairs, let $z:\left(A, A_{0}\right) \rightarrow\left(E, E_{0}\right)$ be a partial section of $p$, let $w:\left(B, B_{0}\right) \rightarrow\left(E, E_{0}\right)$ be a partial lifting of $f$ to $p$.

Definition 2.1.1. Let $\left[\left(X, X_{0}\right) ; p\right]$ be the set of homotopy classes of sections of $p$.

Definition 2.1.2. Let $\left[\left(Y, Y_{0}\right) ; p\right]_{f}$ be the set of homotopy classes of liftings of $f$ to $p$.

Definition 2.1.3. Let $\left[\left(X, X_{0}\right),\left(A, A_{0}\right) ; p\right]^{z}$ be the set of $\operatorname{rel}\left(A, A_{0}\right)$ homotopy classes of sections of $p$ which extend $z$. If $p$ has a standard section, say $s:\left(X, X_{0}\right) \rightarrow\left(E, E_{0}\right)$, then we shall always presume (unless otherwise stated) that $z=s \mid\left(A, A_{0}\right)$ and suppress $z$ in the notation.

Definition 2.1.4. Let $\left[\left(Y, Y_{0}\right),\left(B, B_{0}\right) ; p\right]_{f}^{w}$ be the set of $\operatorname{rel}\left(B, B_{0}\right)$ homotopy classes of liftings of $f$ to $p$ which extend $w$. If $p$ has a standard section, $s$, presume that $w=s f \mid\left(B, B_{0}\right)$ and suppress $w$ in the notation.

The following lemma will be important later.

Lemma 2.1.5. If $\left(\left(X, X_{0}\right),\left(A, A_{0}\right)\right)$ is a C.W. pair of pairs, $p$ is a pair fibration over $\left(X, X_{0}\right),\left\{z_{t}\right\}$ is a homotopy of partial sections of $p$ over $\left(A, A_{0}\right)$, there is a one-to-one correspondence $\left[\left(X, X_{0}\right),\left(A, A_{0}\right) ; p\right]^{z_{0}} \cong\left[\left(X, X_{0}\right),\left(A, A_{0}\right) ; p\right]^{z_{1}}$ such that, whenever $\left\{g_{t}\right\}$ is a homotopy of sections of $p$ which extends $\left\{z_{t}\right\}$, $\left[g_{0}\right]$ (the homotopy class containing $g_{0}$ ) corresponds to $\left[g_{1}\right]$.

Proof. By induction on the skeleta of $X$. We omit the details.

2.2. Fiberwise suspensions. Choose standard spheres and balls $S^{N-1} \subset B^{N}$ for all $N \geqslant 0$ (where $S^{-1}$ is empty) such that $S^{0} * S^{N-1}=S^{N}$ and $S^{0} * B^{N}$ $=B^{N+1}$ (where $*=$ join). Let each sphere have a South pole and a North pole, preserved under inclusion $S^{N} \subset S^{N+1}$. The South pole will be considered to be the base point of each sphere.

If $p: E \rightarrow X$ is a fibration, let $S^{N} p: S_{X}^{N} E \rightarrow X$, where $S_{X}^{N} E=S^{N}{ }^{*} E$, the fiberwise join of $p: E \rightarrow X$ with the trivial fibration $X \times S^{N} \rightarrow X$. We give $S^{N}{ }^{*} E$ the strong topology, thus insuring that $S^{N} p$ is a fibration [5]. Note that $\stackrel{X}{S}^{0} p=p$, and that for $N \geqslant 1, S^{N} p$ has two standard sections, the South polar and the North polar, denoted $s_{0}$ and $s_{1}$. If only one section is needed, $s_{0}$ shall be used. Note also that $S p=S^{1} p$ is the fiberwise two point suspension of $p$.

Now, for any $N \geqslant 0$, let $P^{N} S^{N} p: P_{X}^{N} S_{X}^{N} \rightarrow X$ be defined as follows. For each $x \in X$, let $\left(P^{N} S^{N} p\right)^{-1}\{x\}$ be the set of all maps $\sigma: B^{N} \rightarrow\left(S^{N} p\right)^{-1}\{x\}$ such that $\sigma \mid S^{N-1}$ is simply the identity (recall that each fiber of $S^{N} p$ contains a copy of $S^{N-1}$ ). Let $P_{X}^{N} S_{X}^{N} E$ then have the topology of a subspace of $\left(S_{X}^{N} E\right)^{B^{N}}$ with the compact-open topology. We can define a fiber-preserving inclusion $P_{X}^{N} S_{X}^{N} E \subset P_{X}^{N+1} S_{X}^{N+1} E$ by identifying each $\sigma \in P_{X}^{N} S_{X}^{N} E$ with $S^{0} * \sigma$.

Now if $M \geqslant 0, \quad N \geqslant 1$, let $\Omega^{M} S^{N} p: \Omega_{X}^{M} S_{X}^{N} E \rightarrow X$ be given by 
$\left(\Omega^{M} S^{N} p\right)^{-1}\{x\}=\Omega^{M}\left(S^{N} p\right)^{-1}\{x\}$, the $M$-fold loop space, which we take to be the set of maps $\left(B^{M}, S^{M-1}\right) \rightarrow\left(\left(S^{N} p\right)^{-1}\{x\}, s_{0} x\right)$, for any $x \in X$. We use the compact open topology for $\Omega_{X}^{M} S_{X}^{N} E$, and again there is an inclusion $\Omega_{X}^{M} S_{X}^{M} E$ $\subset \Omega_{X}^{M+1} S_{X}^{N+1} E$.

The above constructions generalize naturally to pair fibrations. If $p:\left(E, E_{0}\right) \rightarrow\left(X, X_{0}\right)$ is a pair fibration, we define:

$$
\begin{aligned}
S^{N} p:\left(S_{X}^{N} E, S_{X_{0}}^{N} E_{0}\right) & \rightarrow\left(X, X_{0}\right), \\
P^{N} S^{N} p:\left(P_{X}^{N} S_{X}^{N} E, P_{X_{0}}^{N} S_{X_{0}}^{N} E_{0}\right) & \rightarrow\left(X, X_{0}\right), \\
\Omega^{M} S^{N} p:\left(\Omega_{X}^{M} S_{X}^{N} E, \Omega_{X_{0}}^{M} S_{X_{0}}^{N} E_{0}\right) & \rightarrow\left(X, X_{0}\right) .
\end{aligned}
$$

2.3. Groups and affine groups of sections and liftings. Henceforth in this section, let $\left(\left(X, X_{0}\right),\left(A, A_{0}\right)\right)$ be a C.W. pair of pairs, and $p:\left(E, E_{0}\right) \rightarrow$ $\left(X, X_{0}\right)$ a pair fibration.

Theorem 2.3.1. If $N \geqslant 1, M \geqslant 1$, then $\left[\left(X, X_{0}\right),\left(A, A_{0}\right) ; \Omega^{M} S^{N} p\right]$ is a group. Furthermore, it is Abelian if $M \geqslant 2$.

We omit the proof of 2.3.1: simply follow, fiberwise, the usual proof that [ $X, A ; \Omega^{M} Y$ ] is a group, Abelian if $M \geqslant 2$, if $Y$ is any pointed space.

The affine structure. Let $N \geqslant 1$, and fix a partial section of $P^{N} S^{N} p$ over $\left(A, A_{0}\right)$. We proceed to define a ternary operation, $\tau$, on $\left[\left(X, X_{0}\right),\left(A, A_{0}\right) ; P^{N} S^{N} p\right]^{2}$.

Let $U$ and $L$ be the upper and lower hemispheres, respectively, of $\partial B^{N}$. Let $B_{1}^{N}, B_{2}^{N}$, and $B_{3}^{N}$ be copies of $B^{N}$, with upper and lower hemispheres $U_{1}, L_{1}, U_{2}$, etc. Now choose a homeomorphism $\phi: B^{N} \rightarrow W=\left(B_{1}^{N} \cup B_{2}^{N} \cup\right.$ $\left.B_{3}^{N}\right) / \sim$, where " $\sim$ " identifies $U_{1}$ with $U_{2}$ and $L_{2}$ with $L_{3}$, and $\phi: L \cong$ $L_{1}, \phi: U \cong U_{3}$. (Note that $\phi$ has degree 1 in homology onto $B_{1}^{N}$ and $B_{3}^{N}$, and -1 onto $B_{2}^{N}$.) Now every section of $P^{N} S^{N} p$ may be thought of as a map $X \times B^{N} \rightarrow S_{X}^{N} E$ satisfying the appropriate conditions. If $g_{1}, g_{2}$, and $g_{3}$ are sections of $P^{N} S^{N} p$, we define a new section of $P^{N} S^{N} p$ called $g_{1} \wedge g_{2}^{-1} \wedge g_{3}$, by commutativity of the following diagram for each $i=1,2,3$ :

$$
\begin{array}{ccc}
X \times B^{N} & g_{1} \wedge g^{-1} \wedge g_{3} & S_{X}^{N} E \\
\downarrow_{1 \times} \times \phi & & \uparrow g_{i} \\
X \times W & \supset X \times B_{i}^{N} \cong \quad X \times B^{N}
\end{array}
$$

Now if $g$ is any section, $g \wedge g^{-1} \wedge g$ is canonically homotopic to $g$ itself. (To see this, let $\chi: W \rightarrow B^{N}$ be the "triple folding" map obtained by identifying each $B_{i}^{N}$ with $B^{N}$. Now choose a rel $S^{N-1}$ homotopy of $\chi \circ \phi: B^{N}$ $\rightarrow B^{N}$ with the identity; this gives a homotopy of $g$ with $g \wedge g^{-1} \wedge g=1_{X}$ $X(\chi \circ \phi)$. This homotopy is functorial with respect to maps of pair fibrations.) Thus, since $z \wedge z^{-1} \wedge z$ is homotopic to $z$, we obtain, by Lemma 
2.1.5, a one-to-one correspondence

$$
\xi:\left[\left(X, X_{0}\right),\left(A, A_{0}\right) ; P^{N} S^{N} p\right]^{z \wedge z^{-1} \wedge z} \cong\left[\left(X, X_{0}\right),\left(A, A_{0}\right) ; P^{N} S^{N} p\right]^{z} \text {. }
$$

Now define $\tau\left(\left[g_{1}\right],\left[g_{2}\right],\left[g_{3}\right]\right)=\left[g_{1}\right]\left[g_{2}\right]^{-1}\left[g_{3}\right]=\xi\left[g_{1} \wedge g_{2}^{-1} \wedge g_{3}\right]$.

THEOREM 2.3.2. (I) Under $\tau,\left[\left(X, X_{0}\right),\left(A, A_{0}\right) ; P^{N} S^{N} p\right]^{z}$ is an affine group. If $N>2$, it is an Abelian affine group. (II) If $\left[\left(X, X_{0}\right),\left(A, A_{0}\right) ; P^{N} S^{N} p\right]^{2}$ is nonempty, its left action group is $\left[\left(X, X_{0}\right),\left(A, A_{0}\right) ; \Omega^{N} S^{N} p\right]$.

Proof. It is a routine exercise to show that $\tau$ is associative and satisfies cancellation, and thus is an affine structure. To obtain (II), consider the map $\eta: B^{N} \rightarrow Y=\left(B_{1}^{N} \cup B_{2}^{N}\right) / \sim \cong S^{N}$ where " " identifies $S_{1}^{N-1}$ with $S_{2}^{N-1}$, and $\eta$ maps all of $S^{N-1}$ onto the common South pole, and is of degree 1 onto $B_{1}^{N}$, degree -1 onto $B_{2}^{N}$. Then let $\left[g_{1}\right]\left[g_{2}\right]^{-1}=\left[g_{1} \wedge g_{2}^{-1}\right]$, where, for $i=1$ or 2, the following diagram commutes:

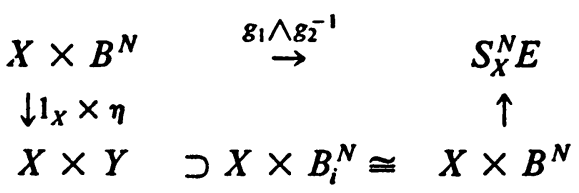

Clearly $g_{1} \wedge g_{2}^{-1}$ is a section of $\Omega^{N} S^{N} p$, and verification of the remaining details is routine. (Hint: $z \wedge z^{-1}$ is canonically homotopic to a trivial section.)

The commutativity of $\tau$ in the case $N>2$ now follows from 2.3.1, and we are done with the proof of 2.3.2.

In certain cases which turn out to be important later there is a geometric definition of the affine structure. Suppose that $U_{1}$ and $U_{2}$ are open subsets of $X$ such that $U_{1} \cup U_{2}=X$, and suppose that $z$ is a partial section of $P^{N} S^{N} p$ $\operatorname{over}\left(A, A_{0}\right)$.

Proposition 2.3.3. If $g_{1}$ and $g_{2}$ are sections of $\Omega^{M} S^{N} p$, for $M>1, N>1$, such that $g_{1}$ and are both trivial on $\left(A, A_{0}\right)$, and such that $g_{1} \mid U_{2}$ is trivial and $g_{2} \mid U_{1}$ is trivial, let $g_{3}$ be the section which agrees with $g_{1}$ on $U_{1}$, with $g_{2}$ on $U_{2}$, and is trivial elsewhere. Then $\left[g_{1}\right]\left[g_{2}\right]=\left[g_{3}\right]$.

Proof. It is clear that $g_{3}$ is homotopic to $g_{1} \wedge g_{2}$ on $X-U_{1}$ and also on $X-U_{2}$; they are both trivial on $U_{1} \cap U_{2}$. Homotopies on the two closed sets may easily be chosen, and do not interfere.

Proposition 2.3.4. If $g_{1}, g_{2}$, and $g_{3}$ are sections of $P^{N} S^{N} p$, for $N>1$, such that all agree with $z$ on $\left(A, A_{0}\right)$, and such that $g_{1}$ agrees with $g_{2}$ outside of $U_{2}$, and $g_{3}$ agrees with $g_{2}$ outside of $U_{3}$, let $g_{4}$ be the section where, for all $x \in X$, 


$$
h_{4}(x)= \begin{cases}h_{1}(x) & \text { if } x \notin U_{2}, \\ h_{2}(x) & \text { if } x \in U_{1} \cap U_{2}, \\ h_{3}(x) & \text { if } x \notin U_{1} .\end{cases}
$$

Then $\left[h_{1}\right]\left[h_{2}\right]^{-1}\left[h_{3}\right]=\left[h_{4}\right]$.

Proof. Note that $h_{1} \wedge h_{2}^{-1} \wedge h_{3}$ and $h_{4}$ are homotopic, albeit via different standard homotopies, on each of the two sets $U_{1}$ and $U_{2}$. By choosing a Urysohn function $X \rightarrow I$ which is 0 on the complement of $U_{2}$ and 1 on the complement of $U_{1}$, these homotopies can be made to blend smoothly into one another over $U_{1} \cap U_{2}$, since on that region $h_{1}, h_{2}, h_{3}$, and $h_{4}$ all agree.

2.4. Equivalence of classes of sections. Let $\left(\left(X, X_{0}\right),\left(A, A_{0}\right)\right)$ be a C.W. pair of pairs, and let $p:\left(E, E_{0}\right) \rightarrow\left(X, X_{0}\right)$ and $p^{\prime}:\left(E^{\prime}, E_{0}^{\prime}\right) \rightarrow\left(X, X_{0}\right)$ be pair fibrations. Let $\gamma:\left(E, E_{0}\right) \rightarrow\left(E^{\prime}, E_{0}^{\prime}\right)$ be a fiber map, and $z:\left(A, A_{0}\right) \rightarrow\left(E, E_{0}\right)$ a partial section of $p$. Finally, define $z^{\prime}=\gamma \cdot z$, a partial section of $p^{\prime}$ over $\left(A, A_{0}\right)$. We have an obvious function, induced by composition with $\gamma$ :

$$
\gamma_{\#}:\left[\left(X, X_{0}\right),\left(A, A_{0}\right) ; p\right]^{z} \rightarrow\left[\left(X, X_{0}\right),\left(A, A_{0}\right) ; p^{\prime}\right]^{z^{\prime}} \text {. }
$$

Under certain conditions on the homotopy of the fibers, $\gamma_{\#}$ is one-to-one or onto.

Let $E_{x}=p^{-1}\{x\}$, for any $x \in X$, and let $\left(E_{0}\right)_{x}=E_{x} \cap E_{0}$. Similarly, let $E_{x}^{\prime}=\left(p^{\prime}\right)^{-1}\{x\}$ and $\left(E_{0}^{\prime}\right)_{x}=E_{x}^{\prime} \cap E_{0}^{\prime}$.

THEOREM 2.4.1. Suppose that $X / A$ is finite dimensional. Let $n=\operatorname{dim}\left(X /\left(X_{0}\right.\right.$ $\cup A)$ ), and $n_{0}=\operatorname{dim}\left(X_{0} / A_{0}\right)$. (I) Suppose that, for all $x \in\left(X-\left(X_{0} \cup A\right)\right)$, $\gamma_{*}: \pi_{k}\left(E_{x}\right) \rightarrow \pi_{k}\left(E_{x}^{\prime}\right)$ is one-to-one and onto for all $0 \leqslant k<n$ and onto for $k=n$; and that, for all $x \in\left(X_{0}-A_{0}\right), \gamma_{*}: \pi_{k}\left(E_{0}\right)_{x} \rightarrow \pi_{k}\left(E_{0}^{\prime}\right)_{x}$ is one-to-one and onto for all $0 \leqslant k<n_{0}$, and onto for $k=n_{0}$. Then $\gamma_{\#}$ is onto. (II) Suppose that, for all $x \in\left(X-\left(X_{0} \cup A\right)\right), \gamma_{*}: \pi_{k}\left(E_{x}\right) \rightarrow \pi_{k}\left(E_{x}^{\prime}\right)$ is one-to-one and onto for all $0<k \leqslant n$ and onto for $k=n+1$; and that $\gamma_{*}: \pi_{k}\left(E_{0}\right)_{x} \rightarrow \pi_{k}\left(E_{0}^{\prime}\right)_{x}$ is one-to-one and onto for all $0 \leqslant k \leqslant n_{0}$ and onto for $k=n_{0}+1$ for all $x \in\left(X_{0}-A_{0}\right)$. Then $\gamma_{\#}$ is one-to-one.

Proof. This theorem is simply a pair-fibration version of a well-known result in fibrations. (See, for example, Lemma 2.2 of [8].) The proof is unaltered by the pair nature, since it is done inductively one cell at a time.

Definition 2.4.2. We say that $p$ is $n$-connected if, for all $k \leqslant n, \pi_{k}(E)_{x}=0$ for all $x \in X$ and $\pi_{k}\left(E_{0}\right)_{x}=0$ for all $x \in X_{0}$.

COROLlaRY 2.4.3. If $p$ is n-connected and $\operatorname{dim}(X / A) \leqslant 2 n$, then $\left[\left(X, X_{0}\right)\right.$, $\left.\left(A, A_{0}\right) ; p\right]^{2}$ is an Abelian affine group.

Proof. The inclusion $i$ : $p \subset P^{N} S^{N} p$ induces isomorphism on the homotopy of the fibers up through dimension $2 n$, and epimorphism in dimension $2 n+1$ 
(since each fiber of $P^{N} S^{N} p$ is of the same homotopy type as the $N$-fold loop space of the $N$-fold suspension of the corresponding fiber of $p$ ). Thus, by 2.4.1, [( $\left.\left.X, X_{0}\right),\left(A, A_{0}\right) ; p\right]^{z} \cong\left[\left(X, X_{0}\right),\left(A, A_{0}\right) ; P^{N} S^{N} p\right]^{i z}$ for all $N$. Apply 2.3.2, and we are done.

We remark that the Abelian affine structure on $\left[\left(X, X_{0}\right),\left(A, A_{0}\right) ; p\right]^{2}$, under the hypotheses of 2.4 .3 , can be alternatively defined in manner described in 2.3.4. Verification is trivial.

2.5. The obstruction theory. Let $\left(\left(X, X_{0}\right),\left(A, A_{0}\right)\right)$ be a C.W. pair of pairs, $p:\left(E, E_{0}\right) \rightarrow\left(X, X_{0}\right)$ a pair fibration, and $z$ a partial section of $p$ over $\left(A, A_{0}\right)$.

DEFINITION 2.5.1. For any integer $i$, let

$$
H^{i}\left(\left(X, X_{0}\right),\left(A, A_{0}\right) ; p\right)=\lim _{N \rightarrow \infty}\left[\left(X, X_{0}\right),\left(A, A_{0}\right) ; \Omega^{N-i} S^{N} p\right],
$$

the direct limit. Write $H^{i}\left(\left(X, X_{0}\right) ; p\right)$ if $A$ is empty.

We remark in passing that $H^{*}(; p)$ is a cohomology theory in a certain sense; and satisfies a version of the Eilenberg-Steenrod axioms. In particular, if $f:\left(\left(Y, Y_{0}\right),\left(B, B_{0}\right)\right) \rightarrow\left(\left(X, X_{0}\right),\left(A, A_{0}\right)\right)$ is a map of C.W. pairs of pairs, there is an induced homomorphism

$$
f^{*}: H^{i}\left(\left(X, X_{0}\right),\left(A, A_{0}\right) ; p\right) \rightarrow H^{i}\left(\left(Y, Y_{0}\right),\left(B, B_{0}\right) ; f^{-1} p\right),
$$

and if $q:\left(F, F_{0}\right) \rightarrow\left(X, X_{0}\right)$ is another pair fibration and $\gamma:\left(E, E_{0}\right) \rightarrow\left(F, F_{0}\right)$ a fiber preserving map, there is an induced homomorphism

$$
\gamma_{\#}: H^{i}\left(\left(X, X_{0}\right),\left(A, A_{0}\right) ; p\right) \rightarrow H^{i}\left(\left(X, X_{0}\right),\left(A, A_{0}\right) ; q\right) .
$$

REMARK 2.5.2. If $p$ is $n$-connected and $\operatorname{dim}(X / A)<2 n$, then $\left[\left(X, X_{0}\right)\right.$, $\left.\left(A, A_{0}\right) ; p\right]^{z}$ (an Abelian affine group, by 2.4.3), if nonempty, has $H^{0}\left(\left(X, X_{0}\right)\right.$, $\left.\left(A, A_{0}\right) ; p\right)$ as its action group.

Thus, classification of sections of a pair fibration, under suitable dimensional restrictions, reduces to algebraic computation of a cohomology group. In the next section, we shall show how $H^{*}\left(\left(X, X_{0}\right),\left(A, A_{0}\right) ; p\right)$ can be attacked by a familiar spectral sequence technique.

The single obstruction. We now consider the question of whether $z$ can be extended to a section of $p$. Recall that $S p$ has two sections, $s_{0}$ and $s_{1}$, the South and North polar sections. Now $S_{X} E$ may be obtained from $E \times I$ by collapsing the ends in the appropriate manner (although with the strong, not the quotient topology). Let $u_{t} x=[z x, t] \in S_{A} E$ for all $x \in A$. By 2.1.5, $\left\{u_{t}\right\}$ gives a one-to-one correspondence:

$$
\text { б: }\left[\left(X, X_{0}\right),\left(A, A_{0}\right) ; S p\right]^{s_{1} \mid A} \cong\left[\left(X, X_{0}\right),\left(A, A_{0}\right) ; S p\right] .
$$

Definition 2.5.3. Let $\gamma(p)=\sigma\left[s_{1}\right] \in\left[\left(X, X_{0}\right),\left(A, A_{0}\right) ; S p\right]$, the primitive single obstruction to section of $p$ extending $z$.

Definition 2.5.4. Let $\Gamma(p) \in H^{1}\left(\left(X, X_{0}\right),\left(A, A_{0}\right) ; p\right)$ be the image of $\gamma(p)$ in the direct limit, the single obstruction to section of $p$ extending $z$. 
THEOREM 2.5.5. (I) There exists a section of $p$ extending $z \Rightarrow \gamma(p)=0 \Rightarrow$ $\Gamma(p)=0$. (II) Suppose that $p$ is n-connected and $\operatorname{dim}(X / A) \leqslant 2 n+1$. Then $\Gamma(p)=0 \Rightarrow \gamma(p)=0 \Rightarrow$ there exists a section of $p$ extending $z$.

Proof. (I) If $g$ is an extension of $z$, the homotopy $\left\{u_{t}\right\}$ can obviously be extended to a homotopy of $s_{0}$ with $s_{1}$, hence $\sigma\left[s_{1}\right]=\left[s_{0}\right]=0$. The second implication is obvious. (II) If $\Gamma(p)=0$, then $\gamma(p)=0$ by 2.4.1. Thus, there is a homotopy between $s_{0}$ and $s_{1}$, extending $\left\{u_{t}\right\}$, hence a section of $P S p$ extending $i z$. By 2.4.1, $i_{\#}:\left[\left(X, X_{0}\right),\left(A, A_{0}\right) ; p\right]^{2} \rightarrow\left[\left(X, X_{0}\right),\left(A, A_{0}\right) ; P S p\right]^{i z}$ is onto, and we are done.

The single difference. Suppose now that $\left\{z_{t}\right\}$ is a homotopy of partial sections of $p$ over $\left(A, A_{0}\right)$. Suppose that $g_{0}$ and $g_{1}$ are sections of $p$ extending $z_{0}$ and $z_{1}$, respectively. Recalling the construction in the proof of 2.3.2, we have $\left(i g_{1}\right) \wedge\left(i g_{0}\right)^{-1}$, a section of $\Omega P p$. Now that section extends $\left(i z_{1}\right) \wedge$ $\left(i z_{0}\right)^{-1}$ which is homotopic (by continuously varying the first index from 1 to $0)$ to $\left(i z_{0}\right) \wedge\left(i z_{0}\right)^{-1}$, which in turn is canonically null-homotopic. Thus, using 2.1.5, we can define:

Definition 2.5.6. Let $\delta\left(g_{0}, g_{1} ;\left\{z_{t}\right\}\right) \in\left[\left(X, X_{0}\right),\left(A, A_{0}\right) ; \Omega P p\right]$, the primitive single difference be the element represented by $\left(i g_{1}\right) \wedge\left(i g_{0}\right)^{-1}$. If $z_{t}=z$ for all $t$, write $\delta\left(g_{0}, g_{1} ; z\right)$.

Definition 2.5.7. Let $\Delta\left(g_{0}, g_{1} ;\left\{z_{t}\right\}\right) \in H^{0}\left(\left(X, X_{0}\right),\left(A, A_{0}\right) ; p\right)$, the single difference class be the image of $\delta\left(g_{0}, g_{1} ;\left\{z_{t}\right\}\right)$ in the direct limit. Write $\Delta\left(g_{0}, g_{1} ; z\right)$ if $z_{t}=z$ for all $t$.

THEOREM 2.5.8 (I) The homotopy $\left\{z_{t}\right\}$ can be extended to a homotopy of $g_{0}$ with $g_{1} \Rightarrow \delta\left(g_{0}, g_{1} ;\left\{z_{t}\right\}\right)=0 \Rightarrow \Delta\left(g_{0}, g_{1} ;\left\{z_{t}\right\}\right)=0$. (II) Suppose that $p$ is $n$-connected for some $n$, and $\operatorname{dim}(X / A) \leqslant 2 n+1$. Then $\Delta\left(g_{0}, g_{1} ;\left\{z_{t}\right\}\right)=0 \Rightarrow$ $\delta\left(g_{0}, g_{1} ;\left\{z_{t}\right\}\right)=0 \Rightarrow$ there exists a homotopy of $g_{0}$ with $g_{1}$ which extends $\left\{z_{t}\right\}$.

Proof. Similar to that of 2.5.5.

2.6. Obstructions to embedding and isotopy. Much of the following material is from [7]. If $M$ is any manifold, of dimension $n$, let $P M$ be the projective bundle associated with the tangent bundle of $M$, let $R^{*} M=\left(M^{2}-\Delta M\right) / T$, where $T(x, y)=(y, x)$, and let $\mathbf{R}^{*} M=R^{*} M \cup P M$, a manifold with boundary $P M$. Let $\left({ }^{N} \mathbf{R}^{*} M,{ }^{N} P M\right)=\left(\mathbf{R}^{*}\left(M \times R^{N}\right), P\left(M \times R^{N}\right)\right)$, and let $\left({ }^{\infty} \mathbf{R}^{*} M,{ }^{\infty} P M\right)$ be the obvious union with the weak topology. The inclusion $\left(\mathbf{R}^{*} M, P M\right) \subset\left({ }^{\infty} \mathbf{R}^{*} M,{ }^{\infty} P M\right)$ we replace by a fibration of pairs in a standard manner: Let $Y_{M}$ be the space of all paths $\sigma: I \rightarrow{ }^{\infty} \mathbf{R}^{*} M$ such that $\sigma(1) \in \mathbf{R}^{*} M$ and let $Z_{M}$ be the space of all paths $\sigma: I \rightarrow{ }^{\infty} P M$ such that $\sigma(1) \in P M$, and let $\zeta_{M}:\left(Y_{M}, Z_{M}\right) \rightarrow\left({ }^{\infty} \mathbf{R}^{*} M,{ }^{\infty} P M\right)$ be evaluation at 0 , an $(n-2)$-connected pair fibration.

Now let $V$ be a compact manifold of dimension $k$, and let $f: V \rightarrow M$ be a differentiable map. Choose, once and for all, an embedding i: $V \subset R^{\infty}$. Let 
$F=\mathbf{R}^{*}(f, \mathbf{i}):\left(\mathbf{R}^{*} V, P V\right) \rightarrow\left({ }^{\infty} \mathbf{R}^{*} M,{ }^{\infty} P M\right)$. We now have a diagram (basically diagram (3.2-1) of [7], with slightly changed notation):

$$
\begin{array}{ccc} 
& \left(Y_{M}, Z_{M}\right) \\
& & \downarrow \zeta_{M} \\
\left(\mathbf{R}^{*} V, P V\right) & \stackrel{F}{\rightarrow} & \left({ }^{\infty} \mathbf{R}^{*} M,{ }^{\infty} P M\right)
\end{array}
$$

Now define a function $\phi:[V \subset M]_{f} \rightarrow\left[\left(\mathbf{R}^{*} V, P V\right) ; \zeta_{M}\right]_{F}$ as follows. If $\left\{f_{t}\right\}$ is an embedding homotopy of $f$, i.e., $f_{0}=f$ and $f_{1}$ is an embedding, let $\Phi\left\{f_{t}\right\}:\left(\mathbf{R}^{*} V, P V\right) \rightarrow\left(Y_{M}, Z_{M}\right)$ be defined as follows. For any $0<u<1$ and any $\mathbf{r} \in \mathbf{R}^{*} V, \Phi\left\{f_{t}\right\}(\mathbf{r})(u)=\mathbf{R}^{*}\left(f_{u},(1-u) \mathbf{i}\right)(\mathbf{r})$. Simply then let $\phi\left[f_{t}\right]$ be the homotopy class containing $\Phi\left\{f_{t}\right\}$. It follows directly ${ }^{2}$ from Theorems 3.3.1 and 3.3.2 of [7] that:

THEOREM 2.6.1. (I) If $2 n \geqslant 3(k+1), \phi$ is onto. (II) If $2 n>3(k+1), \phi$ is one-to-one.

Definition 2.6.2. For any integer $i$, let $H^{i}(f)=H^{i}\left(\left(\mathbf{R}^{*} V, P V\right) ; F^{-} \zeta_{M}\right)$.

Definition 2.6.3. Let $\Gamma(f)=\Gamma(F) \in H^{1}(f)$, the single obstruction to homotopy of $f$ with an embedding.

DEFINITION 2.6.4. Suppose that $\left\{f_{t}^{1}\right\}$ and $\left\{f_{t}^{2}\right\}$ are both $e$-homotopies of $f$. Then let $\Delta\left(\left\{f_{t}^{1}\right\},\left\{f_{t}^{2}\right\}\right)=\Delta\left(\Phi\left\{f_{t}^{1}\right\}, \Phi\left\{f_{t}^{2}\right\}\right) \in H^{0}(f)$.

Now, from 2.5.2, 2.5.5, and 2.5.8, we immediately have:

THEOREM 2.6.5. If $2 n>3(k+1),[V \subset M]_{f}$ is an Abelian affine group, and, if nonempty, it has action group $H^{0}(f)$.

THEOREM 2.6.6. If $f$ is homotopic to an embedding, $\Gamma(f)=0$. The converse holds if $2 n \geqslant 3(k+1)$.

THEOREM 2.6.7. Suppose $\left\{f_{t}^{1}\right\}$ and $\left\{f_{t}^{2}\right\}$ are embedding homotopies of $f$. If they are isotopic, $\Delta\left(\Phi\left\{f_{t}^{1}\right\}, \Phi\left\{f_{t}^{2}\right\}\right)=0$. The converse holds if $2 n>3(k+1)$.

Finally, the following remarks will show how $[V \subset M]_{f}$, up to isomorphism, depends only on the homotopy class of $f$. Let $f_{t}: V \rightarrow M$, $0<t<1$, be any homotopy, where $f_{0}$ and $f_{1}$ are differentiable. Let $\left\{f_{t}\right\}^{*}:$ [ $V$ $\subset M]_{f_{1}} \rightarrow[V \subset M]_{f_{0}}$ be the function, where, if $\left\{g_{t}\right\}$ is an $e$-homotopy of $f_{1}$, $\left\{f_{t}\right\}^{*}\left[g_{t}\right]=\left[h_{t}\right]$, where $h_{t}=f_{2 t}$ or $g_{2 t-1}$, depending on the value of $t$. Clearly $\left\{f_{t}\right\}^{*}$ is one-to-one and onto, since $\left\{f_{1-t}\right\}^{*}$ is its two-sided inverse.

THEOREM 2.7.1. If $2 n>3(k+1),\left\{f_{t}\right\}^{*}$ is an isomorphism of affine groups.

PRoof. Applying the polyhedral covering homotopy property (pair version)

\footnotetext{
${ }^{2}$ Theorems 3.3.1 and 3.3.2 of [7] are correct as stated in that paper, but the proofs are invalid, as has been kindly pointed out by the referree of this paper. A correction is contained here, as an appendix, 88.
} 
of $\zeta_{M}$, one may easily show that, for any $0 \leqslant u \leqslant 1,\left(i_{u}\right)^{*}:\left[\left(\mathbf{R}^{*} V \times I, P V \times\right.\right.$ $\left.I) ; \zeta_{M}\right]_{F p} \rightarrow\left[\left(\mathbf{R}^{*} V, P V\right) ; \zeta_{M}\right]_{F}$ is an isomorphism, where $i_{u}(\mathbf{r})=(\mathbf{r}, u)$ for all $\mathbf{r} \in \mathbf{R}^{*} V$ and $p(\mathbf{r}, u)=\mathbf{r}$. Very simply, checking definitions, one can see that $\left\{f_{t}\right\}^{*}=\left(i_{0}\right)^{*}\left(\left(i_{1}\right)^{*}\right)^{-1}$, and we are done.

In a similar manner, we shall write $\left\{f_{t}\right\}^{*}: H^{i}\left(f_{1}\right) \cong H^{i}\left(f_{0}\right)$ for any integer $i$. We leave the details to the reader.

3. A spectral sequence. In this section, let $\left(\left(X, X_{0}\right),\left(A, A_{0}\right)\right)$ be a fixed C.W. pair of pairs, and let $\zeta:\left(E, E_{0}\right) \rightarrow\left(X, X_{0}\right)$ be a pair fibration. We consider the problem of enumeration of sections of $\zeta$ from a spectral sequence viewpoint.

All pair fibrations shall be over $\left(X, X_{0}\right)$.

3.1. Homotopy sheaves. Let $\zeta$ have a section $s$. We define $\pi_{k}(\zeta, s)$, the $k$ th homotopy sheaf of $\zeta$, to be the sheaf over $X$ determined by a presheaf $\Pi$, where, if $U \subset X$ is open and $U_{0}=U \cap X_{0}, \Pi(U)=\left[\left(U, U_{0}\right) ; \Omega^{k} \zeta\right]$. Hence, for any $x \in X$,

$$
\pi_{k}(\zeta, s)_{x}= \begin{cases}\pi_{k}\left(E_{x}, s x\right) & \text { if } x \in X-X_{0}, \\ \pi_{k}\left(\left(E_{0}\right)_{x}, s x\right) & \text { if } x \in X_{0} .\end{cases}
$$

The total space of $\pi_{k}(\zeta, s)$ need not be Hausdorff.

If each $E_{x}$ and each $\left(E_{0}\right)_{x}$ is $k$-simple, $\pi_{k}(\zeta)$ can be defined regardless of choice (or even existence) of a section. We leave the details to the reader. (Hint: it is sufficient to define $\Pi(U)$ for $U$ contractible.)

Note that the concept of homotopy sheaf is simply a generalization of the usual local system of coefficients for a fibration.

Without any restrictions on $\zeta$, we can always define $\pi_{k}^{S}(\zeta)=$ $\operatorname{Lim}_{N \rightarrow \infty} \pi_{k}\left(\Omega^{N} S^{N \zeta}\right)$, the $k$ th stable homotopy sheaf.

Let $G$ be a sheaf over $X$. We say that $\zeta$ is an Eilenberg Mac Lane pair fibration of type $(G, n)$ (we will write $\zeta=k(G, n))$ if:

(i) $\zeta$ has a section,

(ii) $\pi_{n}(\zeta)=G$,

(iii) $\pi_{k}(\zeta)=0$ for all $k \neq n$.

It is important to note that $G$ cannot simply be any sheaf of groups, Abelian if $n \geqslant 2$. Only for $G$ satisfying special conditions will $k(G, n)$ exist.

In this context, Theorem 2.5.1 of [7] becomes:

THEOREM 3.1.1. If $\zeta=k(G, n)$ for some sheaf of Abelian groups $G$, then $\left[\left(X, X_{0}\right),\left(A, A_{0}\right) ; \zeta\right] \cong H^{n}(X, A ; G)$.

3.2. The fiber of a pair-fibration map. Let $\zeta^{\prime}:\left(E^{\prime}, E_{0}^{\prime}\right) \rightarrow\left(X, X_{0}\right)$ be another pair fibration. We say that $\gamma$ is a pair fibration map, and simply write $\gamma: \zeta \rightarrow \zeta^{\prime}$, if $\gamma:\left(E, E_{0}\right) \rightarrow\left(E^{\prime}, E_{0}^{\prime}\right)$ si simply a fiber-preserving map. Suppose that $\zeta$ has a section $s$. Then $s^{\prime}=\gamma s$ is a section of $\zeta^{\prime}$. We define a pair 
fibration $\phi[\gamma]:\left(F, F_{0}\right) \rightarrow\left(X, X_{0}\right)$, called the fiber of $\gamma$, as follows: for each $x \in X, F_{x}$ is the usual homotopy theoretic fiber of $E_{x} \rightarrow E_{x}^{\prime}$, that is, $F_{x}=$ $\left\{(e, \sigma) \mid e \in E_{x}, \sigma: I \rightarrow E_{x}^{\prime}, \sigma(0)=s^{\prime} x, \sigma(1)=\gamma e\right\}$, and $\left(F_{0}\right)_{x}$ is the homotopy theoretic fiber of $\left(E_{0}\right)_{x} \rightarrow\left(E_{0}^{\prime}\right)_{x}$. Let $\lambda: \phi[\gamma] \rightarrow \zeta$ be given by $\lambda(e, \sigma)=e$, and let $\iota: \Omega \zeta^{\prime} \rightarrow \phi[\gamma]$ be the obvious inclusion.

Identifying $\Omega \phi[\gamma]$ with $\phi[\Omega \gamma]$ in the obvious way, we have:

LEMMA 3.2.1. The following long sequence is exact:

$$
\begin{aligned}
\cdots & \rightarrow\left[\left(X, X_{0}\right),\left(A, A_{0}\right) ; \Omega \zeta\right]^{(\Omega \gamma} \stackrel{()_{*}}{\rightarrow}\left[\left(X, X_{0}\right),\left(A, A_{0}\right) ; \Omega \zeta^{\prime}\right] \\
& \stackrel{\iota}{\rightarrow}\left[\left(X, X_{0}\right),\left(A, A_{0}\right) ; \phi[\gamma]\right] \\
& \stackrel{\lambda_{\#}}{\rightarrow}\left[\left(X, X_{0}\right),\left(A, A_{0}\right) ; \zeta\right] \stackrel{\gamma=}{\rightarrow}\left[\left(X, X_{0}\right),\left(A, A_{0}\right) ; \zeta^{\prime}\right]
\end{aligned}
$$

Proof. Since the definition of fiber the natural definition for the category of pair-fibrations over $\left(X, X_{0}\right)$, the details of the proof are routine and obvious.

Similarly, we have:

LEMMA 3.2.2. The following sequence of homotopy sheaves is exact:

$$
\cdots \stackrel{\gamma_{\#}}{\rightarrow} \pi_{k}\left(\Omega \zeta^{\prime}\right)=\pi_{k-1}\left(\zeta^{\prime}\right) \stackrel{\iota \#}{\rightarrow} \pi_{k}(\phi[\gamma]) \stackrel{\lambda_{\#}}{\rightarrow} \pi_{k}(\zeta) \stackrel{\gamma_{\#}}{\rightarrow} \pi_{k}\left(\zeta^{\prime}\right) \rightarrow \cdots \cdot
$$

\subsection{The homotopy killing constructions.}

The strong topology double mapping cylinder. Let $A, B$, and $C$ be topological spaces, and $\alpha: B \rightarrow A, \beta: B \rightarrow C$ maps. Let $A \cup_{\alpha}(B \times I) \cup_{\beta} C$ be the space obtained from $A \cup(B \times I) \cup C$ by identifying $(x, 0)$ with $\alpha x$ and $(x, 1)$ with $\beta x$ for all $x \in B$; but with the strong topology, not the quotient topology. Neighborhoods of points in $B \times(0,1)$ are as usual in the product topology, while if $a \in A$, a basic neighborhood of $a \in W$ is of the form $U \cup\left[\alpha^{-1} U \times[0, \varepsilon)\right]$ for $\varepsilon>0$ and $U$ a neighborhood of $a$ in $A$. Neighborhoods along $C$ are similar. Equivalently, the strong topology is the strongest topology such that all of the following obvious projections are continuous:

$$
\begin{aligned}
A \cup_{\alpha}(B \times I) \cup_{\beta} C & \rightarrow I, \\
A \cup_{\alpha}(B \times[0,1)) & \rightarrow A, \\
(B \times(0,1]) \cup_{\beta} C & \rightarrow C . \\
B \times(0,1) & \rightarrow B .
\end{aligned}
$$

Now suppose that $a:\left(A, A_{0}\right) \rightarrow\left(X, X_{0}\right), b:\left(B, B_{0}\right) \rightarrow\left(X, X_{0}\right), c:\left(C, C_{0}\right) \rightarrow$ $\left(X, X_{0}\right), \alpha:\left(B, B_{0}\right) \rightarrow\left(A, A_{0}\right)$, and $\beta:\left(B, B_{0}\right) \rightarrow\left(C, C_{0}\right)$ are all pair fibrations.

LEMMA 3.3.1. $a \cup_{\alpha}(b \times I) \cup_{\beta} c:\left(A \cup_{\alpha}(B \times I) \cup_{\beta} C, A_{0} \cup_{\alpha}\left(B_{0} \times I\right)\right.$ $\left.\cup_{\beta} C_{0}\right) \rightarrow\left(X, X_{0}\right)$ is a pair fibration. 
Proof. Omit. See Hall [5] for the proof of a special case, the fiberwise strong topology join.

Now suppose that, for all $n \geqslant 1$, we have pair fibrations $a_{n}, b_{n}, c_{n}, \alpha_{n}$, and $\beta_{n}$ (as in the hypotheses of 3.3.1), such that $a_{n+1}=a_{n} \cup_{\alpha_{n}}\left(b_{n} \times I\right) \cup_{\beta_{n}} c_{n}$ for all $n$. Let $a_{\infty}=\cup a_{n}$, with the weak topology.

LEMMA 3.3.2. $a_{\infty}$ is a pair fibration.

Proof. Essentially by induction on $n$, mimicking the proof of 3.3.1 at each step. We omit the details.

Let $E_{X}^{S^{n}} \rightarrow X$ be the fibration where, for each $x \in X,\left(E_{X}^{S^{n}}\right)_{x}=\left(E_{x}\right)^{S^{n}}$, the space of maps $S^{n} \rightarrow E_{x}$, with the compact open topology. Let $e: E_{X}^{S^{n}} \times S^{n} \rightarrow$ $E$ be the evaluation map, and let $K_{X}^{n} E=E \cup \cup_{e}\left(E_{X}^{S^{n}} \times B^{n+1}\right)$, with the strong topology. (Define as follows: Since $B^{n+1}$ is the cone over $S^{n}, K_{X}^{n} E=$ $E \cup_{e}\left(E_{X}^{S^{n}} \times S^{n} \times I\right) \cup_{\pi} E_{X}^{S^{n}}$, the strong topology double mapping cylinder, where $\pi$ is projection.) Now let $K^{n \zeta:}\left(K_{X}^{n} E, K_{X_{0}}^{n} E_{0}\right) \rightarrow\left(X, X_{0}\right)$; by 3.2.1, a pair fibration.

THEOREM 3.3.3. (I) If $k<n, i_{\#}: \pi_{k}(\zeta) \cong \pi_{k}\left(K^{n \zeta}\right)$. (II) $\pi_{n}\left(K^{n \zeta}\right)=0$.

Proof. We first need a lemma.

LEMMA 3.3.4. If $Z$ is any simplicial complex of dimension less than or equal to $n$, and if $Y$ is any space, then $[Z ; Y] \rightarrow\left[Z ; Y \cup \cup_{e}\left(Y^{S^{n}} \times B^{n+1}\right)\right]$ is onto.

Proof of lemma. Let $* \in B^{n+1}$ be its center. If $f: Z \rightarrow Y \cup_{e}\left(Y^{S^{n}} \times\right.$ $\left.B^{n+1}\right), f$ can be deformed slightly so that its image does not intersect $Y^{S^{n}} \times\{*\}$. The complement of that subset collapses to $Y$, and we are done.

Returning to the proof of 3.3.3, we have immediately from the lemma that $\pi_{k}(\zeta) \rightarrow \pi_{k}\left(K^{n} \zeta\right)$ is onto for $k \leqslant n$ and one-to-one for $k<n$. Finally, $\pi_{n}(\zeta)$ $\rightarrow \pi_{n}\left(K^{n \zeta}\right)$ is the zero map, since the construction attaches an $(n+1)$-cell to every possible map of $S^{n}$ to every fiber.

Definition 3.3.5. For $0 \leqslant n \leqslant m$, let $K^{n, m} \zeta$ be defined inductively by $K^{n, m} \zeta=K^{n} \zeta$, and $K^{n, m+1} \zeta=K^{m+1}\left(K^{n, m} \zeta\right)$. Finally, let $\mathbf{K}^{n \zeta}=$ $\cup_{m} K^{n, m} \zeta$.

THEOREM 3.3.6. (I) If $k<n, \pi_{k}(\zeta) \rightarrow \pi_{k}\left(\mathbf{K}^{n \zeta}\right)$. (II) If $k \geqslant n, \pi_{k}\left(\mathbf{K}^{n \zeta}\right)=0$.

Proof. Direct from 3.3.3.

3.4. The spectral sequence determined by a resolution. Suppose that $\zeta$ has a section. We say that the following commutative diagram of sectioned pair fibrations and maps

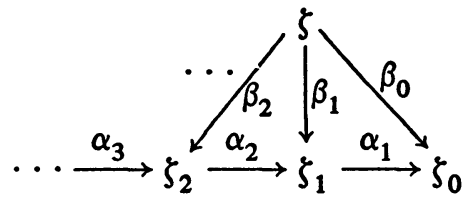


is a resolution if, for each integer $n$, there exists an integer $N(n)$ such that $\left(\alpha_{m}\right)_{\#}: \pi_{k}\left(\zeta_{m}\right) \cong \pi_{k}\left(\zeta_{m-1}\right)$ and $\left(\beta_{m}\right)_{\#}: \pi_{k}(\zeta) \cong \pi_{k}\left(\zeta_{m}\right)$ for all $k<n$ and all $m>N(n)$. We say that it is a Postnikov resolution if, for every $n>0$, $\left(\beta_{n}\right)_{\#}: \pi_{k}(\zeta) \approx \pi_{k}\left(\zeta_{n}\right)$ for all $k<n$, and $\pi_{k}\left(\zeta_{n}\right)=0$ for all $k>n$.

THEOREM 3.4.1. If $\zeta$ has a section, $\zeta$ has a Postnikov resolution. Furthermore, if $\gamma: \zeta \rightarrow \zeta^{\prime}, \gamma$ induces a natural map of Postnikov resolutions.

Proof. For every integer $n \geqslant 0$, let $\zeta_{n}=K^{n+1} \zeta$. Let the $\alpha_{i}$ and $\beta_{i}$ be the inclusions. By 3.3.6, we are done.

LeMma 3.4.2. If (3.4-1) is a Postnikov resolution, $\phi\left[\alpha_{n}\right]=k\left(\pi_{n}(\zeta), n\right)$.

Proof. Directly from 3.2.2.

Suppose diagram (3.4-1) is given. We define (taking $\zeta_{q}:\left(X, X_{0}\right) \rightarrow\left(X, X_{0}\right)$ for $q<0)$ :

$$
\begin{aligned}
E_{2}^{p, q} & =\left[\left(X, X_{0}\right),\left(A, A_{0}\right) ; \Omega^{-p} \phi\left[\alpha_{q}\right]\right] \text { for } p \leqslant 0, \\
D_{2}^{p, q} & =\left[\left(X, X_{0}\right),\left(A, A_{0}\right) ; \Omega^{-p \zeta_{q}}\right] \text { for } p \leqslant 0, \\
i_{2} & =\left(\Omega^{-p} \alpha_{q}\right)_{\#}: D_{2}^{p, q} \rightarrow D_{2}^{p, q-1} \text { for } p<0, \\
j_{2} & =\left(\Omega^{-p-1} \iota\right)_{\#}: D_{2}^{p, q} \rightarrow E_{2}^{p+1, q+1} \text { for } p \leqslant-1, \\
k_{2} & =\left(\Omega^{-p} \lambda\right)_{\#}: E_{2}^{p, q} \rightarrow D_{2}^{p, q} \text { for } p \leqslant 0 .
\end{aligned}
$$

By 3.2.1, we now have a bigraded exact couple (although there is an edge problem, since $p$ must not be positive). Furthermore, from 3.1.1 and 3.4.2, we immediately have:

Remark 3.4.3. If (3.4-1) is a Postnikov resolution, $E_{2}^{p, q}=H^{p+q}(X, A$; $\left.\pi_{q}(\zeta)\right)$.

Let $E_{r}^{p, q}, D_{r}^{p, q}, E_{\infty}^{p, q}$, and $D_{\infty}^{p, q}$ be obtained in the usual manner.

THEOREM 3.4.4. If $p \leqslant-1$, the $E_{\infty}^{p, q}$ (for various $q$ ) give a composition series for $\left[\left(X, X_{0}\right),\left(A, A_{0}\right) ; \Omega^{-p} \zeta\right]$, provided $\operatorname{dim}(X / A)<\infty$.

PRoof. Let $G^{p, q}$ be the kernel of $\left(\Omega^{-p} \beta_{q}\right)_{\#}:\left[\left(X, X_{0}\right),\left(A, A_{0}\right) ; \Omega^{-p} \zeta\right] \rightarrow D_{2}^{p . q}$. Thus, $G^{p,-1}=\left[\left(X, X_{0}\right),\left(A, A_{0}\right) ; \Omega^{-p} \zeta\right]$ and, for dimensional reasons, $G^{p, q}=$ 0 for $q$ sufficiently large. By standard spectral sequence arguments, $E_{\infty}^{p . q}=$ $G^{p, q-1} / G^{p, q}$, and we are done.

If $\zeta$ is deloopable, i.e., $\zeta=\Omega \eta$, we can, by constructing a Postnikov resolution for $\eta$, obtain a spectral sequence converging to a composition series for $\left[\left(X, X_{0}\right),\left(A, A_{0}\right) ; \zeta\right]$. If $\zeta$ is infinitely deloopable, the exact couple can be constructed with no restrictions on the indices. Since computation of $H^{i}\left(\left(X, X_{0}\right),\left(A, A_{0}\right) ; \zeta\right)$ for $\operatorname{dim}(X / A)<\infty$ involves classifying sections of $\Omega^{N} S^{i+N \zeta}$ for large $N$, that cohomology theory can also be obtained by a 
spectral sequence, where $E_{2}^{p, q}=H^{p+q}\left(X, A ; \pi_{q}^{S}(\zeta)\right)$. We leave the details to the reader.

4. A geometric interpretation of the affine structure. Let $f: V \rightarrow M$ be a map, where $V$ is a compact manifold of dimension $k$, and $M$ is a manifold of dimension $n$, where $2 n>3(k+1)$. We give a geometric interpretation of the affine group structure of $[V \subset M]_{f}$.

By Theorem 2.7.1, it is sufficient to consider the case where $f$ is actually an embedding. We then consider $[V \subset M]_{f}$ to be an actual Abelian group with identity $[f]$, represented by the constant homotopy.

Let $\left\{f_{t}^{1}\right\},\left\{f_{t}^{2}\right\}$ be $e$-homotopies of $f$ such that

(i) $f_{t}^{1}$ and $f_{t}^{2}$ are differentiable for all $t \in I$.

(ii) For some disjoint closed sets $K_{1}, K_{2} \subset V$, and for all $t \in I$, $f_{t}^{i}$ agrees with $f$ on $V-K_{i}$.

(iii) For any $x_{1} \in K_{1}, x_{2} \in K_{2}, x_{3} \in V-\left(K_{1} \cup K_{2}\right)$, and for any $t, u \in I$; $f_{t}^{1}\left(x_{1}\right), f_{t}^{2}\left(x_{2}\right)$, and $f\left(x_{3}\right)$ are distinct.

Now let $f_{t}^{3}: V \rightarrow M$ be the $e$-homotopy of $f$ such that, for all $x \in V, t \in I$ :

$$
f_{t}^{3}(x)= \begin{cases}f_{t}^{1}(x) & \text { if } x \in K_{1} \\ f_{t}^{2}(x) & \text { if } x \in K_{2} \\ f(x) & \text { otherwise }\end{cases}
$$

THEOREM 4.1. $\left[f_{t}^{1}\right]+\left[f_{t}^{2}\right]=\left[f_{t}^{3}\right]$.

Proof. We need only show that

$$
\left[\iota \Phi\left\{f_{t}^{1}\right\}\right][\iota \Phi\{f\}]^{-1}\left[\iota \Phi\left\{f_{t}^{2}\right\}\right]=\left[\iota \Phi\left\{f_{t}^{3}\right\}\right] \in\left[\left(\mathbf{R}^{*} V, P V\right) ; P S \zeta_{M}\right]_{F},
$$

where $\iota: \zeta_{M} \subset P S \zeta_{M}$. Pick disjoint closed sets $L_{1}, L_{2}$ in $V$ such that $K_{i} \subset$ Int $L_{i}$. Choose continuous functions $\rho_{1}, \rho_{2}: V \rightarrow I$ such that $\rho_{i}(x)=1$ for all $x \in K_{i}$, and 0 for all $x \notin L_{i}$. For each $i=1,2$, or 3 , let $\Phi_{i, u}, 0<u<1$, be the homotopy of liftings of $F$ to $\zeta_{M}$ such that, for all $u, \Phi_{i, u}$ agrees with $\Phi\left\{f_{i}^{i}\right\}$ on $P V$, and such that for all $0<t, u<1$ and all $[x, y] \in R^{*} V$,

$$
\begin{aligned}
\Phi_{i, u}[x, y](t) & =\left[\left(f_{v}^{i}(x),(1-t) \mathbf{i}(x)\right),\left(f_{w}^{i}(y),(1-t) \mathbf{i}(y)\right)\right] \\
& \in R^{*}\left(M \times R^{\infty}\right)={ }^{\infty} R^{*} M,
\end{aligned}
$$

where:

$$
\begin{gathered}
v=t\left(u \rho_{i}(x)+1-u\right) \text { and } \quad w=t\left(u \rho_{i}(y)+1-u\right) \text { if } i=1 \text { or } 2, \\
v=t\left(u \rho_{j}(x)+1-u\right) \quad \text { and } \quad w=t\left(u \rho_{j}(y)+1-u\right) \\
\text { if } i=3, j=1 \text { or } 2, \text { and } \rho_{j}(x) \rho_{j}(y)>0, \\
v=w=t(1-u) \quad \text { if } i=3, \text { and } \rho_{1}(x) \rho_{1}(y)+\rho_{2}(x) \rho_{2}(y)=0 .
\end{gathered}
$$


Note then that $\Phi_{0}^{i}=\Phi\left\{f_{t}^{i}\right\}$ for all $i=1,2$, or 3 , and by 2.3.4, letting $D_{i}=Q\left(L_{i}^{2}\right) \subset \mathbf{R}^{*} V$ for $i=1$ or 2 , we obtain: $\left[\iota \Phi_{1}^{1}\right][\iota \Phi\{f\}]^{-1}\left[\iota \Phi_{1}^{2}\right]=\left[\iota \Phi_{1}^{3}\right]$, and we are done.

Using a general position argument, based on the dimensional restriction, it is possible to show that any two $e$-homotopies of $f$ are respectively isotopic to a pair of $e$-homotopies satisfying (i) through (iii) above. Thus Theorem 4.1 suffices to describe the affine structure on $[V \subset M]_{f}$.

In later calculations, the following result is useful:

THEOREM 4.2. If $f^{\prime}: V \rightarrow M$ is another map such that $f \mid D$ is homotopic to $f^{\prime} \mid D$ for some $D \subset V$ such that $V-D \cong R^{n}$, then $[V \subset M]_{f} \cong[V \subset M]_{f}$, if both are nonempty.

Proof. Without loss of generality, $f$ and $f^{\prime}$ are both embeddings, and $f^{\prime}|D=f| D$. If $\alpha \in[V \subset M]_{f}$, we may, by a general position argument, choose an e-homotopy $\left\{f_{t}\right\}$ of $f$ such that

(i) $\left[f_{t}\right]=\alpha$,

(ii) $f_{t}|(V-D)=f|(V-D)$ for all $t$,

(iii) $f_{t}(D) \cap f^{\prime}(V-D)$ is empty for all $t$.

Now let $\phi(\alpha)=\left[f_{t}^{\prime}\right]$, where, for all $0 \leqslant t \leqslant 1, f_{t}^{\prime}(x)=f_{t}(x)$ if $x \in D, f_{t}^{\prime}(x)=$ $f^{\prime}(x)$ if $x \in(V-D)$. Using 4.1 and standard general position arguments, one may easily show that $\phi:[V \subset M]_{f} \rightarrow[V \subset M]_{f}$, is well defined, and is an isomorphism.

5. The structure of $\pi_{n} \zeta_{M}$. Let $M$ be any connected $n$-manifold. In this section, we explicitly compute the sheaf $\pi_{n} \zeta_{M}$ over ${ }^{\infty} \mathbf{R}^{*} M$, provided $n>6$. (The sheaf $\pi_{n-1} \zeta_{M}$ was computed in [7], provided $n \geqslant 5$; we restate the results here. For $k<n-1, \pi_{k} \zeta_{M}=0$.)

5.1. Building a twisted sheaf. Suppose that $X$ is any path-connected space with basepoint $x_{0}$, and $S$ is a sheaf over $X$ with a local product structure. Let $S_{x}$ be the stalk of $S$ over any $x \in X$. If $a \in \pi_{1} X$, let $\alpha:(I, \partial I) \rightarrow\left(X, x_{0}\right)$ be a loop representing $a$, and let $\tilde{\alpha}: I \times S_{x_{0}} \rightarrow S$ be a map such that $\tilde{\alpha}(t, s) \in$ $S_{\alpha(t)}$ and $\tilde{\alpha}(1, s)=s$ for all $t \in I, s \in S_{x_{0}}$. Let $\langle a\rangle: S_{x_{0}} \rightarrow S_{x_{0}}$ be the automorphism where $\langle a\rangle(s)=\tilde{\alpha}(0, s)$. Let $\mu_{S}: \pi_{1} X \times S_{x_{0}} \rightarrow S_{x_{0}}$ be the left action where $\mu_{S}(a, s)=\langle a\rangle(s)$ for all $a \in \pi_{1} X, s \in S_{x_{0}}$.

LEMMA 5.1.1. Let $X$ be path connected and locally simply connected, with basepoint. Let $G$ be any group and let $\mu: \pi_{1} X \times G \rightarrow G$ be any left action. Then there exists a sheaf $S=S(G, \mu)$, with local product structure over $X$, such that $S_{x_{0}}=G$ and $\mu_{S}=\mu$. Furthermore, $S$ is unique with these properties, up to isomorphism.

Proof. Let $\pi: Y \rightarrow X$ be the universal covering of $X$, and let $\nu: \pi_{1} X \times Y$ $\rightarrow Y$ be the associated (left) action, where $\pi y_{1}=y_{2}$ if and only if $\nu\left(a, y_{2}\right)$ for 
some $a \in \pi_{1} X$. Then let $S(G, \mu)$ be the quotient space of $Y \times G$ obtained by identifying each $(y, g)$ with $(\nu(a, y), \mu(a, g))$ for all $a \in \pi_{1} X$. Trivially, the required conditions are satisfied. The proof of uniqueness is trivial and straightforward, hence we omit it.

5.2. Preliminary definitions. For any topological space $X$, let $\Gamma X$ be the quotient space of $X \times X \times S^{\infty}$ obtained by identifying each $(x, y, a)$ with $T(x, y, a)=(y, x,-a)$. If $f: X_{1} \rightarrow X_{2}$ is any map, let $\Gamma f: \Gamma X_{1} \rightarrow \Gamma X_{2}$ be the obvious map. Note that if $*$ is a single point space, $\Gamma *=p^{\infty}=S^{\infty} / T$. Let Г0: $\Gamma X \rightarrow P^{\infty}$, where $0: X \rightarrow *$ is the collapsing map. If $* \in X$ is a basepoint, let $j=\Gamma i: P^{\infty} \rightarrow \Gamma X$, where $i: * \rightarrow X$ is the inclusion. Let $\pi: X \times X \times S^{\infty}$ $\rightarrow \Gamma X$ denote the 2-1 covering, and, by a slight abuse of notation, let $\pi: X \times X \rightarrow \Gamma X$ denote the (equivalent to the covering) inclusion, where $\pi(x, y)=[x, y, *], * \in S^{\infty}$ a basepoint. We may consider $X \times P^{\infty} \subset \Gamma X$; if $x \in X$ and $a \in S^{\infty}$, identify $(x,[a])$ with $[x, x, a]$.

Let $X$ be any topological space with basepoint, and let $\pi_{k}=\pi_{k} X$, written multiplicatively if $k=1$, additively if $k>1$. Let $\gamma_{k}: \pi_{1} \times \pi_{k} \rightarrow \pi_{k}$ be the usual left action, determined by the map $\gamma_{k}: S^{k} \rightarrow S^{1} \vee S^{k}$, where

$$
\gamma_{k}\left[x_{1}, x_{2}, \ldots, x_{k}\right]= \begin{cases}{\left[2 x_{1}\right] \in S^{1}} & \text { if } 0 \leqslant x_{1} \leqslant \frac{1}{2}, \\ {\left[2 x_{1}-1, x_{2}, \ldots, x_{k}\right] \in S^{k}} & \text { if } \frac{1}{2} \leqslant x_{1} \leqslant 1,\end{cases}
$$

where $S^{k}=I^{k} / \partial I^{k}$. We let $x^{a}=\gamma_{k}(a, x)$ for all $a \in \pi_{1}, x \in \pi_{k}$. Let $\alpha_{k}: \pi_{2}$ $\otimes \pi_{k} \rightarrow \pi_{k+1}$, for $k \geqslant 2$, be the Whitehead product, determined by the map $\alpha_{k}: S^{k+1} \rightarrow S^{2} \bigvee S^{k}$, where

$$
\alpha_{k}\left(x_{1}, x_{2}, \ldots, x_{k+2}\right)=\left\{\begin{array}{r}
{\left[x_{1}, x_{2}\right] \in S^{2}} \\
\text { if } x_{i} \in \partial I \text { for some } 3 \leqslant i \leqslant k+2, \\
{\left[x_{3}, \ldots, x_{k+2}\right] \in S^{k} \quad \text { if } x_{i} \in \partial I \text { for } i=1 \text { or } 2,}
\end{array}\right.
$$

where $S^{2}=I^{2} / \partial I^{2}, S^{k}=I^{k} / \partial I^{k}$, and $S^{k+1}=\partial I^{k+2}$.

If $X$ is a manifold and $x \in \pi_{k}$, let $w_{k} x \in Z_{2}$ be the value of $f^{*} w_{k} X \in$ $H^{k}\left(S^{k} ; Z_{2}\right)$ (the Stiefel-Whitney class), where $f: S^{k} \rightarrow X$ represents $x$. If $a \in \pi_{1}$, let $(-1)^{a}=1$ if $w_{1} a=0,-1$ otherwise.

Again, if $X$ is a manifold, and if $a \in \pi_{1}, a^{2}=1$ (the identity), let $f_{a}: P^{2} \rightarrow$ $X$ be a map which sends the generator of $\pi_{1} P^{2}$ to $a$, and elt $\tilde{a}=\left[f_{a} s\right] \in \pi_{2}$, where $s: S^{2} \rightarrow P^{2}$ is the covering map onto the real projective plane. Let $k_{a}$ be an integer such that the vector bundles $f_{a}^{-1} \tau$ and $k_{a} h$ are stably equivalent, where $\tau$ is the tangent bundle of $X$ and $h$ is the canonical line bundle over $P^{2}$. Note that the pair $\left(k_{a}, \tilde{a}\right)$ has indeterminacy (because of the choice of $f_{a}$ ) $(4 Z \oplus 0)+\operatorname{Im} \chi_{a}$, where $\chi_{a} x=\left(m w_{2} x, x+x^{a}\right)$ for all $x \in \pi_{2}$, where $m: Z_{2}$ $\rightarrow Z_{4}$ is the monomorphism. (Hint: recall that $\tilde{K}^{0}\left(P^{2}\right)=Z_{4}$ )

Let $G$ and $N$ be groups, and let $\varphi: G \rightarrow$ Aut $N$ be any homomorphism. Let 
$N \times_{\varphi} G$ be the semidirect product. Specifically, as a set, $N \times_{\varphi} G$ is the Cartesian product $N \times G$, with the operation $\left(n_{1}, g_{1}\right)\left(n_{2}, g_{2}\right)=$ $\left(n_{1} \varphi\left(g_{1}\right) n_{2}, g_{1} g_{2}\right)$. Note that if $\varphi$ is trivial, $N \times_{\varphi} G$ is simply the direct sum. We always have $G \subset N \times{ }_{\varphi} G$ and $N \triangleleft N \times{ }_{\varphi} G$, where we identify $N$ and $G$ with $N \times\{1\}$ and $\{1\} \times G$, respectively.

For any (multiplicatively written) group $G$, let $Z G$ be the group ring of $G$, which we represent as finite formal sums of elements of $G$. Thus, $G \subset Z G$.

5.3. Structure of $\pi_{n-1} \zeta_{M}$ and $\pi_{n} \zeta_{M}$. Now let $X={ }^{\infty} \mathbf{R}^{*} M$, and $X_{0}={ }^{\infty} P M$. Once and for all, fix basepoints $* \in M$ and $* \in S M$ over $*$, and let $* \in P M$ also denote the image of $*$. Let $\pi_{k}=\pi_{k} M$, written multiplicatively if $k=1$, additively otherwise. For consistency of notation, we introduce a group $T_{2}=\{1, m\}$, a multiplicative group of two elements. Let $H_{2}=\{0, \eta\} \simeq Z_{2}$, the stable 1-stem in the homotopy of spheres, which we treat as a $Z_{2}$-module. For any group $G$, let $\Delta G \subset G \oplus G$ be the diagonal.

LEMMA 5.3.1. (I) $\pi_{1} X=\left(\pi_{1} \oplus \pi_{1}\right) \times_{\varphi} T_{2}$, where $\varphi(m)(a, b)=(b, a)$ for all $a, b \in \pi_{1}$. (II) $\pi_{1} X_{0}=\Delta \pi_{1} \oplus T_{2}$, and $i: X_{0} \subset X$ induces the inclusion of groups described in 5.2.

Proof. Let $\iota: M \times R^{\infty} \rightarrow R^{\infty}$ be any embedding, and let $\rho: \operatorname{R} R^{\infty} \rightarrow S^{\infty}$ be the equivalent retraction, determined by $\rho(u, v)=\|u-v\|^{-1}(u-v)$ for all $(u, v) \in R R^{\infty}$. Let $\psi=\left(p_{1}^{2} Q, \rho \mathbf{R} \iota\right):\left(\mathbf{R}\left(M \times R^{\infty}\right), S\left(M \times R^{\infty}\right)\right) \rightarrow(M$ $\left.\times M \times S^{\infty}, \Delta M \times S^{\infty}\right)$ which is equivariantly a homotopy equivalence of pairs, by an elementary obstruction theory argument. Passing to quotient spaces under the involution $T$, we have that the pair $\left(X, X_{0}\right)$ is of the homotopy type of $\left(\Gamma M, M \times P^{\infty}\right)$.

We have a partially split exact sequence:

$$
\begin{array}{cccccc}
1 \rightarrow & \pi_{1} M^{2} & \rightarrow & \pi_{1} \Gamma M & \stackrel{c_{\#}}{\rightleftarrows} & \pi_{1} P^{\infty}
\end{array} \rightarrow 1
$$

A simple deck-transformation argument then verifies that $\pi_{1} M$ is the desired semi-direct product. (II) follows trivially.

Now let $\theta_{M}: Y \rightarrow X$ and $\rho_{M}: Z \rightarrow X_{0}$ be the fibrations which (as functions) agree with the pair fibration $\zeta_{M}$.

LEMMA 5.3.2. (I) If $n>5, \pi_{n-1} \theta_{M}=S\left(Z \pi_{1}, \mu^{\prime}\right)$, where

(i) $\mu^{\prime}((b, c, 1), a)=(-1)^{c} b a c^{-1}$,

(ii) $\mu^{\prime}((b, c, m), a)=(-1)^{n}(-1)^{a c} b a^{-1} c^{-1}$ for all $a, b, c \in \pi_{1}$.

(II) If $n>6, \pi_{n} \theta_{M}=S\left(Z_{1} \otimes\left(\pi_{2} \oplus H_{2}\right), \mu\right)$, where

(i) $\mu((b, c, 1), a \otimes(x, \lambda \eta))=(-1)^{c} b a c^{-1} \otimes\left(x^{c}, \lambda \eta\right)$,

(ii) $\mu((b, c, m), a \otimes(x, \lambda \eta))=(-1)^{n+1}(-1)^{a c} b a^{-1} c^{-1} \otimes\left(x^{c^{-1} a},(\lambda+\right.$ $\left.w_{2} x\right) \eta$ ) for all $a, b, c \in \pi_{1}, x \in \pi_{2}$, and $\lambda \in Z_{2}$. 
We postpone the proof.

Identify $Z$ with $Z\{1\} \subset Z \pi_{1}$. Let $\nu^{\prime}:\left(\left(Z \Delta \pi_{1}\right) \oplus T_{2}\right) \times Z \rightarrow Z$ and $\nu:\left(\left(Z \Delta \pi_{1}\right) \oplus T_{2}\right) \times\left(Z \otimes H_{2}\right) \rightarrow Z \otimes H_{2}$ be the restrictions of $\mu^{\prime}$ and $\mu$, respectively.

LEMMA 5.3.3. (I) If $n \geqslant 5, \pi_{n-1} \rho_{M}=S\left(Z, \nu^{\prime}\right)$. (II) If $n \geqslant 6, \pi_{n} \rho_{M}=S(Z$ $\left.\otimes H_{2}, v\right)$.

We leave the proof to the reader. (Hint: the fiber of $\rho_{M}$ is $S^{n-1}$.)

One final lemma completes the description of $\pi_{n-1} \zeta_{M}$ and $\pi_{n} \zeta_{M}$.

LEMma 5.3.4. If $\pi_{k} \rho_{M}$ is a subsheaf of $\left(\pi_{k} \theta_{M}\right) \mid X_{0}$, then $\pi_{k} \zeta_{M}$ is the subsheaf of $\pi_{k} \theta_{M}$ whose stalks over $X_{0}$ agree with those of $\pi_{k} \theta_{M}$, and whose stalks over $X-X_{0}$ agree with those of $\pi_{k} \rho_{M}$.

Proof. Follows directly from the definition of $\pi_{k} \zeta_{M}$.

5.4. Proof of Lemma 5.3.2. Part (I) of 5.3.2 is simply Lemmas 3.4.2, 3.4.4, and 3.4.5 of [7]. (However, we have replaced the right actions of that paper by left actions.)

Now we saw in the proof of 3.4.2 of [7] that the fiber of $\theta_{M}$ is the fiber $\Phi$ of the inclusion $\tilde{M}^{00} \subset \tilde{M}$, where $\tilde{M}$ is the universal covering space of $M$, and $\tilde{M}^{00}$ is the universal covering space of $M^{0}=M-\{*\}$. A straightforward Serre spectral sequence argument reveals that

(i) $\pi_{n-1}(\Phi)=Z \pi_{1}$,

(ii) $\pi_{n}(\Phi)=Z \pi_{1} \otimes\left(\pi_{2} \oplus H_{2}\right)$,

where composition with $\eta: S^{n} \rightarrow S^{n-1}$ is represented by $\otimes \eta$, and where, for each $a \in \pi_{1}$ and $x \in \pi_{2}, a \otimes x$ is represented by the map $\phi$ in the following homotopy commutative diagram, where $S^{2} \stackrel{\chi}{\rightarrow} \tilde{M}^{00} \subset \tilde{M} \rightarrow M$ represents $x$ :

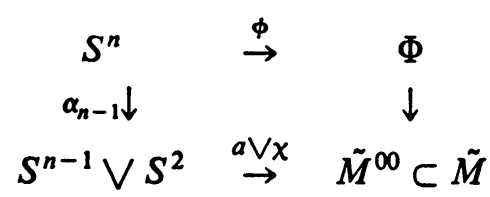

The reader can easily verify that (II) need only be checked for the following five special cases (where $a, b, c \in \pi_{1} ; x \in \pi_{2}$ ):

(i) $((b, 1,1), a \otimes \eta)=b a \otimes \eta$,

(ii) $((1,1, m), a \otimes \eta)=a^{-1} \otimes \eta$,

(iii) $((b, 1,1), a \otimes x)=b a \otimes x$,

(iv) $((1, c, 1), 1 \otimes x)=(-1)^{c} c^{-1} \otimes x^{c}$,

(v) $((1,1, m), 1 \otimes x)=(-1)^{n+1} \otimes\left(\left(w_{2} x\right) \eta+x\right)$.

Now (i), (ii) follow immediately from (I), by composition with $\eta$, while the proof of (iii) involves (I), together with mere straightforward checking of maps. 
To prove (iv), let $\gamma: I \rightarrow M$ be a loop representing $c$. Let $\psi_{t}: S^{2} \rightarrow M$, $0 \leqslant t \leqslant 1$, be a homotopy such that $\psi_{1}(*)=\gamma(t)$ for all $t$, and $\psi_{1}$ represents $x$. Then $\psi_{0}$ represents $x^{c}$.

We briefly introduce a general construction. If $Y$ is any pointed space, $B \subset Y$, and if $f:\left(I^{k}, \partial I^{k}\right) \rightarrow(Y, B)$ is a map such that $f(*)=*$ for some $* \in I^{k}$, let $\langle f\rangle: S^{k-1}=\partial I^{k} \rightarrow F(F=$ homotopy theoretic fiber of the inclusion $B \subset Y)$ be defined as follows: let $\rho: C S^{k-1} \cong I^{k}(C X=$ cone over $X)$ and let $\langle f\rangle(v)=\sigma_{v} \in F \subset Y^{I}$, where $\sigma_{v}(t)=f(\rho[v, t])$. By a slight modification, it is not necessary to assume $B \subset Y$, only $B \rightarrow Y$.

Returning to the proof, choose a homotopy $\theta_{t}:\left(I^{n}, S^{n-1}\right) \rightarrow\left(\tilde{M}^{2}, \tilde{R} M\right)$, where $\tilde{R} M$ is the universal covering space of $R M=M^{2}-\Delta M$, such that:

(i) $p_{2} \theta_{t}(v)=\gamma(t)$ for all $t \in I$,

(ii) $\left\langle\theta_{1}\right\rangle$ represents $1 \in Z \pi_{1}=\pi_{n-1}(\Phi)$,

where $p_{2}: \tilde{M}^{2} \rightarrow \tilde{M}$ is projection to the second factor. Clearly $\left\langle\theta_{0}\right\rangle$ represents $(-1)^{c} c^{-1} \in Z \pi_{1}=\pi_{n-1}(\Phi)$. Now, for all $t \in I$, we have a homotopy commutative diagram:

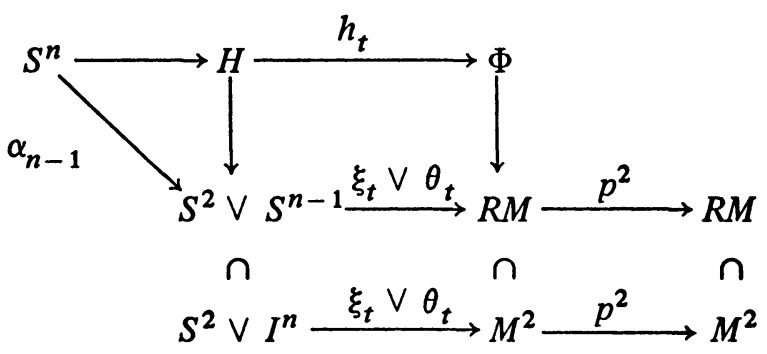

where $p: \tilde{M} \rightarrow M$ is the covering map, $p^{2} \xi_{t}=i_{1} \psi_{t}$ for all $t, \xi_{1}(*)=*$, and $\beta$ is chosen independent of $t$. By definition, $\left[\beta h_{1}\right]=1 \otimes x$ and $\mu((1, c, 1), 1 \otimes x)$ $=\left[\beta h_{0}\right]=(-1)^{c}\left(c^{-1} \otimes x^{c}\right)$, and (iv) is verified.

To prove (v), let $g: R^{n} \rightarrow M$ be a coordinate patch such that $g(0)=*$. Let $\kappa:\left(I^{n}, S^{n-1}\right) \rightarrow\left(R^{n}, R^{n}-\{0\}\right)$ be the obvious orientation preserving equivalence. Then $\langle g \kappa\rangle= \pm 1 \in Z \subset Z \pi_{1}=\pi_{n-1}(\Phi)$. We alter $g$ if necessary to insure that $\langle g \kappa\rangle=1$.

Let $(S M)_{*}$ be the fiber of $S M \rightarrow M$ over *. Choose a homeomorphism $\iota: S^{n-1} \rightarrow(S M)_{*}$ such that $\langle\lambda\rangle=1 \in \pi_{n-1}(\Phi)$, where $\lambda:\left(I^{n}, S^{n-1}\right) \rightarrow$ $\left(M^{2}, \mathbf{R} M\right)$ is chosen such that $\lambda\left(I^{n}\right)=* \in M^{2}$, and $\lambda \mid S^{n-1}=\iota$. When convenient, we shall identify $S^{n-1}$ with $(S M)_{*}$.

Let $r: E \rightarrow S^{2}$ be the $S^{n-1}$ bundle which is the pullback of $S M \rightarrow M$, where $\gamma: S^{2} \rightarrow M$ classifies $x \in \pi_{2}$, and let $j: S^{2} \rightarrow E$ be a section of $r$. Let $e: I^{2} \times S^{n-1} \rightarrow E$ be a map such that the following diagram commutes: 


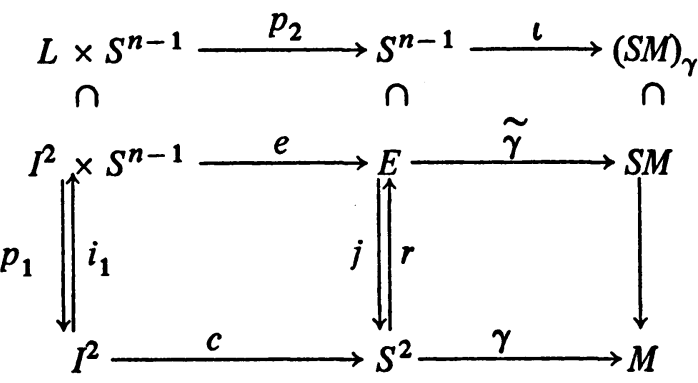

where $p_{i}$ is the projection on the $i$ th factor, $c$ collapses $\partial I^{2}$ to $* \in S^{2}$, $L=\{1\} \times I \cup I \times \partial I \subset I^{2}$, and $i_{1}$ is inclusion along the first factor. Let $y_{t}: I^{2} \rightarrow M^{2}, 0 \leqslant t \leqslant 1$, be the homotopy such that

$$
y_{t}(u, v)= \begin{cases}(\gamma[1-u, v], *) & \text { if } 0 \leqslant u \leqslant t, \\ (\gamma[1-t, v], \gamma[u-t, v]) & \text { if } t \leqslant u \leqslant 1 .\end{cases}
$$

Let $W=\left(S^{1} \times S^{n-1}\right) \cup\left(I^{2} \times\{*\}\right) \subset I^{2} \times S^{n-1}$, where $S^{1}=\partial I^{2}$, and choose a homotopy $\tilde{y}_{t}: W \rightarrow \mathbf{R} M, 0 \leqslant t \leqslant 1$, such that $\tilde{y}_{t} \mid S^{1} \times S^{n-1}=\tilde{\gamma} e\left(J_{t}\right.$ $\times 1)$ for all $t$, where $J_{t}: S^{1} \rightarrow I^{2}$ is given by $J_{t}(u, v)=(1-t u, v)$ for all $(u, v) \in S^{1} \subset I^{2}$; and such that $Q \tilde{y}_{t} \mid I^{2} \times\{*\}$ and $y_{t} p_{1} \mid I^{2} \times\{*\}$ are homotopic, rel $S^{1} \times\{*\}$, for all $t$; where $Q: \mathbf{R} M \rightarrow M^{2}$ is the usual quotient map.

The following diagram illustrates the next portion of the argument.

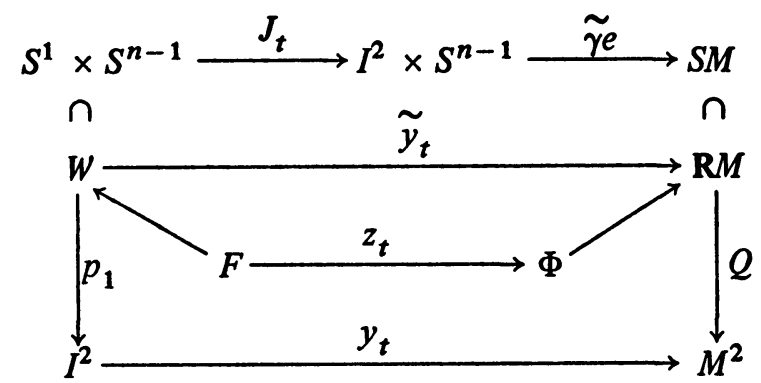

Let $z_{t}: F \rightarrow \Phi, 0 \leqslant t \leqslant 1$, be the homotopy induced by $\left\{y_{t}\right\},\left\{\tilde{y}_{t}\right\}$ (where $F=$ fiber of $p_{1}: W \rightarrow I^{2}$ ). Let $i_{2}: S^{n-1} \subset W$ be inclusion along the second factor, and let $\omega=\left(d_{\#}\right)^{-1}\left[\alpha_{n-1}\right] \in \pi_{n} W=\pi_{n} F$, where $d: W \rightarrow S^{2} \vee S^{n-1}$ is the map which collapses $S^{1} \times\{*\} \subset W$. Clearly $\left(z_{0}\right)_{\#}=(-1)^{n} \mu((1,1, m), 1$ $\otimes x)$; it remains to show only that $\left(z_{1}\right)_{\#} \omega=-(1 \otimes x)+\left(w_{2} x\right) 1 \otimes \eta$.

Note that $\tilde{y}_{1}: S^{1} \times\{*\} \rightarrow j(*) \in(S M)_{*} \subset \mathbf{R} M$, thus $y^{*}: S^{2} \rightarrow \mathbf{R} M$, where $y^{*}[u, v]=y_{1}(u, v, *)$ is well defined. We then have a commutative diagram (where $S^{2}$ is identified with $j S^{2} \subset E$ ): 


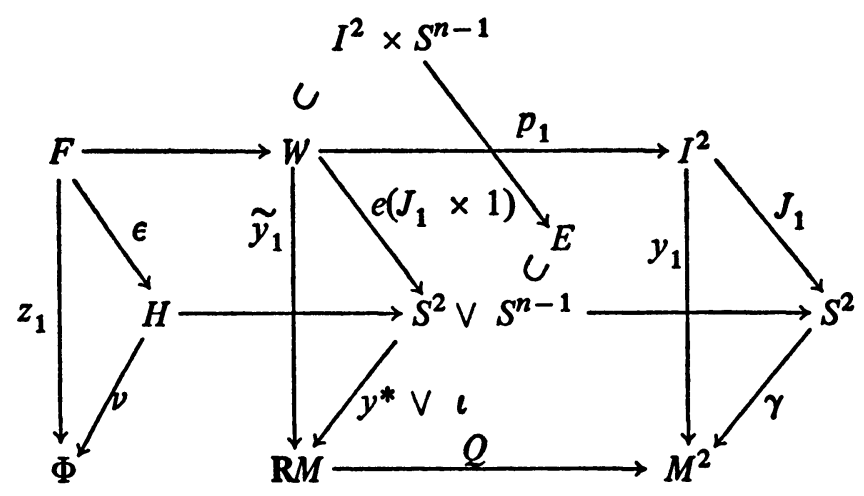

where $\varepsilon, \nu$ are the appropriate maps on the fibers. Now $I^{2} \times S^{n-1}=W$ $\cup_{w} e^{n+1}$, where $w: S^{n} \rightarrow W$ classifies $\omega$, and $E=S^{2} \vee S^{n-1} \cup_{\lambda} e^{n+1}$, where $\lambda=e\left(J_{1} \times 1\right) w$. Now $\mathrm{Sq}^{2} \phi=\gamma^{*} w_{2} M \cup \phi$, and $\phi \cup \sigma=-e^{n+1}$ (since $J_{1}$ has degree -1), where $\phi \in H^{n-1}(E ; Z)$ is the generator and $\sigma \in H^{2}\left(S^{2} ; Z\right)$ is the fundamental class. Thus $[\lambda]=-\left[\alpha_{n-1}\right]+\left(w_{2} x\right)\left[\eta i_{2}\right] \in \pi_{n}\left(S^{2} \vee S^{n-1}\right)$, and we are done.

6. Explicit computation of $\left[S^{k} \subset M^{2 k}\right]_{f}$. In this section, $\left[S^{k} \subset M^{n}\right]_{f}$ is explicitly stated, in terms of generators and relations, where $M$ is any manifold without boundary, $f: S^{k} \rightarrow M$ is any embedding, and $n=2 k+1$, $n \geqslant 2$, or $n=2 k, k \geqslant 3$.

THEOREM 6.0.1. If $k \geqslant 2, n=2 k+1$, then $\left[S^{k} \subset M^{n}\right]_{f}$ is generated by elements $y_{a}$ for all $a \in \pi_{1} M$, subject only to the following relations:

(i) $y_{1}=0$, where $1 \in \pi_{1} M$ is the identity;

(ii) For any $a \in \pi_{1} M, y_{a^{-1}}=(-1)^{k+1}(-1)^{a} y_{a}$.

THEOREM 6.0.2. If $k \geqslant 3, n=2 k$, then $\left[S^{k} \subset M^{n}\right]_{f}$ is generated only by elements:

(i) $z_{a}$ for all $a \in \pi_{1} M$ such that $a^{2}=1$ and $(-1)^{k}(-1)^{a}=1$.

(ii) $y_{a} \otimes u$ for all $a \in \pi_{1} M$ and all $u \in \pi_{2} M \oplus H_{2}$, where $H_{2}=\{0, \eta\}$ is the stable 1-stem in the homotopy of spheres.

And is subject only to the relations:

(iii) $y_{a} \otimes u+y_{a} \otimes v=y_{a} \otimes(u+v)$ for any $a \in \pi_{1} M, u, v \in \pi_{2} M \oplus H_{2}$.

(iv) $y_{1} \otimes \eta=0$.

(v) $z_{1}=0$.

(vi) $y_{a} \otimes \eta=y_{a^{-1}} \otimes \eta$ for any $a \in \pi_{1} M$.

(vii) $y_{a} \otimes x+(-1)^{a}(-1)^{k} y_{a^{-1}} \otimes x^{a}+\left(w_{2} x\right) y_{a} \otimes \eta=0$ for all $a \in \pi_{1} M$, $x \in \pi_{2} M$.

(viii)

$$
y_{a} \otimes \tilde{a}+\left(\left(\begin{array}{c}
k-3 \\
2
\end{array}\right)+\left(\begin{array}{c}
2 k-k_{a} \\
2
\end{array}\right)\right) y_{a} \otimes \eta=0
$$


for all $a \in \pi_{1} M$ such that $a^{2}=1$ and $(-1)^{a}(-1)^{k}=-1$.

(ix)

$$
2 z_{a}=y_{a} \otimes \tilde{a}+\left(\left(\begin{array}{c}
2 k-k_{a} \\
2
\end{array}\right)+\left(\begin{array}{c}
k-3 \\
2
\end{array}\right)+\left(\begin{array}{l}
k \\
1
\end{array}\right)\right) y_{a} \otimes \eta
$$

for all $a \in \pi_{1} M$ such that $a^{2}=1$ and $(-1)^{a}(-1)^{k}=1$.

Finally, these representations are natural, i.e.;

THEOREM 6.0.3. Let $g: M \subset M^{\prime}$ be an embedding, where $M^{\prime}$ is another manifold. Then (I) If $k \geqslant 2, n=2 k+1, g_{\#} y_{a}=y_{g_{*} a}$ for all $a \in \pi_{1} M$. (II) If $k>3, n=2 k, g_{\#}\left(y_{a} \otimes x\right)=y_{g_{*} a} \otimes g_{\#} x$ and $g_{\#}\left(y_{a} \otimes \eta\right)=y_{g_{*} a} \otimes \eta$ for all $a \in \pi_{1} M, x \in \pi_{2} M$, and $g_{\#} z_{a}=z_{g_{*} a}$ if $a \in \pi_{1} M, a^{2}=1$, and $(-1)^{a}(-1)^{k}=$ 1.

6.0.1 and 6.0.2 are proved below (6.0.1 was incorrectly stated as Theorem 1.2.1 of [7], the result there is off by a sign), while we leave the proof of 6.0 .3 to the reader. Basically, 6.0 .3 follows from the fact that all of the constructions are natural. Examination of those constructions reveals that $y_{a}$, $y_{a} \otimes x$ and $y_{a} \otimes \eta$ are canonically chosen, while $z_{a}$ has an indeterminacy, as defined below, the group generated by $y_{a} \otimes \tilde{a}, y_{a} \otimes \eta$, and $y_{a} \otimes x+y_{a} \otimes x^{a}$ for all $x \in \pi_{2} M$. By making a once-and-for-all choice in each of the four universal examples (see the proof of 6.1.3), this indeterminacy could possibly be further reduced.

6.1. Proof of 6.0.1 and 6.0.2. Without loss of generality, $f$ may be assumed inessential (cf. 4.2). Now, if $k \geqslant 2, n=2 k+1$, there is only one nonzero $E_{\infty}$ term in the composition series for $\left[S^{k} \subset M\right]_{f}=H^{0}(f)$, namely $E_{\infty}^{0, n-1}$. Now $E_{2}^{0, n-1}=H^{n-1}\left(\mathbf{R}^{*} S^{k} ; F^{-1} \pi_{n-1} \zeta_{M}\right)$, and our result follows immediately from Lemma 6.1.1, below, where $y_{a}$ corresponds to $[a]$ for all $a \in \pi_{1}=\pi_{1} M$.

Suppose now that $k \geqslant 3, n=2 k$. Let $\pi_{1}=\pi_{1} M, \pi_{2}=\pi_{2} M$. The only $E_{2}$ terms which play a role in the computation of $\left[S^{k} \subset M\right]_{f}$ are $E_{2}^{0, n-1}=$ $H^{n-1}\left(\mathbf{R}^{*} S^{k} ; F^{-1} \pi_{n-1} \zeta_{M}\right), E_{2}^{-1, n-1}=H^{n-2}\left(\mathbf{R}^{*} S^{k} ; F^{-1} \pi_{n-1} \zeta_{M}\right)$, and $E_{2}^{0, n}=$ $H^{n}\left(\mathbf{R}^{*} S^{k} ; F^{-1} \pi_{n} \zeta_{M}\right)$; and the only relevant differential is $d_{2}$ is the exact sequence:

$$
E_{2}^{-1, n-1} \stackrel{d_{2}}{\rightarrow} E_{2}^{0, n} \stackrel{\lambda}{\rightarrow} H^{0}(f) \stackrel{\rho}{\rightarrow} E_{2}^{0, n-1} \rightarrow 0 .
$$

Now if $e: 0 \rightarrow A \rightarrow B \rightarrow C \rightarrow 0$ is any short exact sequence of Abelian groups, $e$ determines a homomorphism $\Phi_{2}: K_{2} \rightarrow A / 2 A$, where $K_{2}=\{x \in$ $C \mid 2 x=0\}$, as follows: for $x \in K_{2}, \Phi_{2} x=\lambda^{-1} 2 \rho^{-1} x$. By Theorem 5.1 of [9], knowledge of $\Phi_{2}$ suffices to determine $B$ as an extension of $C$ by $A$ if $2 C=0$. By 6.1.1, $2 E^{0, n-1}=0$; then (for the sequence (6.1-1)) the groups are given by 6.1.1, and $d_{2}$ and $\Phi_{2}$ are given by 6.1.3, and Theorem 6.0.2 follows immediately; where, for $a \in \pi_{1}, x \in \pi_{2}, \lambda[a \otimes x]=y_{a} \otimes x, \lambda[a \otimes \eta]=y_{a} \otimes$ $\eta$; and, if $a^{2}=1$ and $(-1)^{a}(-1)^{k}=1, \rho z_{a}=[a]$. 
Recall that $H_{2}=\{0, \eta\} \cong Z_{2}$.

For any integer $r, k<r \leqslant 2 k$, let $K_{n-1}^{r}=Z \pi_{1}$ and $K_{n}^{r}=Z \pi_{1} \otimes\left(\pi_{2} \oplus\right.$ $\left.H_{2}\right)$. For $r<k$ or $r>2 k$, let $K_{n-1}^{r}=K_{n}^{r}=0$. Define $\delta_{n-1}^{r}: K_{n-1}^{r} \rightarrow K_{n-1}^{r+1}$ and $\delta_{n}^{r}: K_{n}^{r} \rightarrow K_{n}^{r+1}$ for all $r$ as follows: for $k \leqslant r<2 k$, and for $a \in \pi_{1}, x \in \pi_{2}$ :

(i) $\delta_{n-1}^{r}(a)=a+(-1)^{r+k+n+1}(-1)^{a} a^{-1}$,

(ii) $\delta_{n}^{r}(a \otimes x)=a \otimes x+(-1)^{a}(-1)^{r+k+n} a^{-1} \otimes x^{a}+\left(w_{2} x\right) a^{-1} \otimes \eta$,

(iii) $\delta_{n}^{r}(a \otimes \eta)=a \otimes \eta+a^{-1} \otimes \eta$.

We now define $H_{s}^{r}=\operatorname{Ker} \delta_{s}^{r} / \operatorname{Im} \delta_{s}^{r}$ for $s=n-1$ or $n$.

LEMMA 6.1.1. (I) For all $r$, and for $s=n-1$ or $n$,

$$
H^{r}\left(\mathbf{R}^{*} S^{k}, P S^{k} ; F^{-1} \pi_{s} \zeta_{M}\right)=H^{r}\left(\mathbf{R}^{*} S^{k}, P S^{k} ; F^{-1} \pi_{s} \theta_{M}\right)=H_{s}^{r} .
$$

(II) For all $r>k$, and for $s=n-1$ or $n, j^{*}: H_{s}^{r} \rightarrow H^{r}\left(\mathbf{R} S^{k} ; F^{-1} \pi_{s} \zeta_{M}\right)$ is onto, and

$$
\operatorname{Ker} j^{*}= \begin{cases}\left(Z \cap \operatorname{Ker} \delta_{n-1}^{r}\right) / \operatorname{Im} \delta_{n-1}^{r-1} & \text { if } s=n-1, \\ \left(Z \otimes H_{2} \cap \operatorname{Ker} \delta_{n}^{r}\right) / \operatorname{Im} \delta_{n}^{r-1} & \text { if } s=n .\end{cases}
$$

Proof (I). Trivially,

$$
H^{*}\left(\mathbf{R}^{*} S^{k}, P S^{k} ; F^{-1} \pi_{s} \zeta_{M}\right)=H^{*}\left(\mathbf{R}^{*} S^{k}, P S^{k} ; F^{-1} \pi_{s} \theta_{M}\right),
$$

since these two coefficient sheaves differ only on $P S^{k}$.

We now consider $S^{k} \subset R^{k+1}$ to be the unit sphere, and $\pi: S^{k} \rightarrow P^{k}$ the covering map onto real projective $k$-space. For any $x \in P^{k}$, let $[x] \subset R^{k+1}$ be the line through 0 determined by $x$. Let $\nu$ be the $k$-plane bundle over $P^{k}$ such that, for each $x \in P^{k}, \nu_{x}=[x]^{\perp} \subset R^{k+1}$. Clearly, $\pi^{-1} \nu=\tau$, the tangent bundle of $S^{k}$, and $\nu \oplus h=(k+1)$, the trivial $(k+1)$-plane bundle, where $h$ is the canonical line bundle over $P^{k}$ and " $\oplus$ " denotes Whitney sum. Thus $w_{i} \nu=u^{i}$ for all $i$, where $u \in H^{1}\left(P^{k} ; Z_{2}\right)$ is the generator.

For any vector bundle $\xi$, let $E \xi$ and $S \xi$ be the total spaces of the associated disc and sphere bundles, respectively. We construct a commutative diagram of pairs

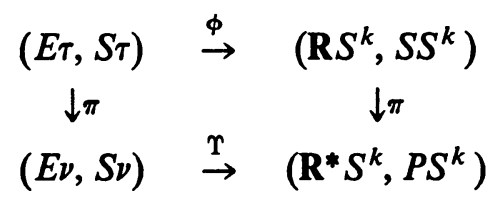

as follows. $E \tau=\left\{(u, v) \in S^{k} \times B^{k+1} \mid u \perp v\right\}$, where $B^{k+1} \subset R^{k+1}$ is the unit ball, while $S S^{k}=S \tau=\left\{(u, v) \in S^{k} \times S^{k} \mid u \perp v\right\}$. For any $(u, v) \in E \tau$, let

$$
\phi(u, v)= \begin{cases}\left(\alpha_{v} v+u, \alpha_{v} v-u\right) \in R S^{k} & \text { if }(u, v) \notin S \tau, \\ (v, u) \in S S^{k} & \text { if }(u, v) \in S \tau,\end{cases}
$$


where $\alpha_{v}=\left(1-\|v\|^{2}\right)^{1 / 2}$, and let $\Upsilon$ be the unique map which makes the diagram commutative. Trivially, $\phi$ and $\Upsilon$ are both homeomorphisms of pairs. Thus $\mathbf{R}^{*} S^{k} / P S^{k} \cong M(\nu)$, the Thom complex of $\nu$, whence $H^{i}\left(\mathbf{R}^{*} S^{k}, P S^{k} ; Z_{2}\right) \cong Z_{2}$ if $k \leqslant i \leqslant 2 k, 0$ otherwise; and $\mathrm{Sq}^{j}: H^{i}\left(\mathbf{R}^{*} S^{k}, P S^{k} ; Z_{2}\right) \rightarrow H^{i+j}\left(\mathbf{R}^{*} S^{k}, P S^{k} ; Z_{2}\right)$ is nonzero if and only if $k \leqslant i \leqslant i+j \leqslant 2 k$ and $\left(\begin{array}{c}i-k-1 \\ j\end{array}\right)=1 \bmod 2\left(\right.$ where we take $\left(\begin{array}{c}-1 \\ j\end{array}\right)=1 \bmod 2$ for all $j$ ). $\mathbf{R} S^{k} / S S^{k}$ may then be considered to have two cells, $e_{1}^{i}$ and $e_{2}^{i}$, in each dimension $k \leqslant i \leqslant 2 k$, and none in other dimensions, such that:

(i) $T e_{1}^{i}=e_{2}^{i}$ for all $i$, where $T: \mathbf{R} S^{k} \rightarrow \mathbf{R} S^{k}$ is the map which exchanges coordinates;

(ii) For all $k \leqslant i<2 k$ and $j=1$ or 2 ,

$$
\delta e_{j}^{i}= \begin{cases}e_{1}^{i+1}+e_{2}^{i+1} & \text { if } i-k \text { is even, } \\ (-1)^{j+1}\left(e_{1}^{i+1}-e_{2}^{i+1}\right) & \text { if } i-k \text { is odd. }\end{cases}
$$

Then $\mathbf{R}^{*} S^{k} / P S^{k}$ has one cell in each dimension $k \leqslant i \leqslant 2 k$, namely $e^{i}=$ $\pi e_{1}^{i}=\pi e_{2}^{i}$, and no cells in other dimensions; and $\delta e^{i}=\left(1+(-1)^{i-k}\right) e^{i+1}$.

Now let $G$ be any Abelian group and $e: G \rightarrow G$ an automorphism of order 2. Recall that $\pi_{1}\left(\mathbf{R}^{*} S^{k}\right) \cong T_{2}=\{1, m\}$, a multiplicative group of order 2 . Let $\mu_{e}: T_{2} \times G \rightarrow G$ be the action where $\mu_{e}(m, x)=e x$ for all $x \in G$, and let $G^{e}=S\left(G, \mu_{e}\right)$, a sheaf over $\mathbf{R}^{*} S^{k} . H^{*}\left(\mathbf{R}^{*} S^{k}, P S^{k} ; G^{e}\right)$ may be considered to be the equivariant cohomology of the pair $\left(\mathbf{R} S^{k}, S S^{k}\right)$ with coefficients in $G$ under the action $\mu_{e}$, specifically, the homology of the chain complex

$$
G \stackrel{d^{k}}{\rightarrow} G \stackrel{d^{k+1}}{\rightarrow} G \rightarrow \cdots \rightarrow G \stackrel{d^{2 k-1}}{\rightarrow} G
$$

where, for $k \leqslant i<2 k, d^{i} x=x+(-1)^{i-k}$ ex for all $x \in G$. Letting $G_{n-1}=$ $Z \pi_{1}$ and $e a=(-1)^{a}(-1)^{n} a^{-1}$ for all $a \in \pi_{1}$, and letting $G_{n}=Z \pi_{1} \otimes\left(\pi_{2} \oplus\right.$ $\left.H_{2}\right)$ and $e(a \otimes(x+\lambda \eta))=\left((-1)^{a}(-1)^{n+1}\right) a^{-1} \otimes x^{a}+a^{-1} \otimes\left(\lambda+w_{2} x\right) \eta$ for all $a \in \pi_{1}, x \in \pi_{2}, \lambda \in Z_{2}$, we have by 5.3.3 that $G_{s}^{e}=F^{-1} \pi_{s} \theta_{M}$ for $s=n-1$ or $n$, and we are done with the proof of (I).

Proof (II). For convenience of notation, we agree to let $S_{s}=F^{-1} \pi_{s} \zeta_{M}$ and $Q_{s}=F^{-1} \pi_{s} \theta_{M}$ for $s=n-1$ or $n$. Let $L_{s} \subset S_{s}$ be the unique maximal subsheaf with a local product structure, specifically, $L_{s}=J_{s}^{e}$, where $J_{n-1}=Z$ $\subset G_{n-1}$ and $J_{n}=Z \otimes H_{2} \subset G_{n}$. Note that $G_{s}=J_{s} \oplus R_{s}$, for suitably chosen $R_{s} \subset G_{s}$, and $T_{2}$ acts independently on each direct summand: it follows that $S_{s}=L_{s} \oplus R_{s}^{e}$, a direct summation of sheaves. We also have that $L_{s} \mid P S^{k}=$ $S_{s} \mid P S^{k}$ and $Q_{s}\left|R^{*} S^{k}=S_{s}\right| R^{*} S^{k}$, and:

$$
\begin{gathered}
L_{n} \cong Z_{2} ; \\
L_{n-1} \cong \begin{cases}Z & \text { if } n \text { is even, } \\
Z^{T} & \text { if } n \text { is odd. }\end{cases}
\end{gathered}
$$

Thus $i^{*}: H^{r}\left(\mathbf{R}^{*} S^{k}, P S^{k} ; L_{s}\right) \rightarrow H^{r}\left(\mathbf{R}^{*} S^{k}, P S^{k} ; S_{s}\right)$ is mono, its image 
exactly what we wish $\operatorname{Ker} j^{*}$ to be. We have a commutative diagram with exact rows (since $H^{i}\left(\mathrm{R}^{*} S^{k} ; L_{s}\right)=0$ for all $i>k$, because $L_{s}$ has a local product structure and $\mathbf{R}^{*} S^{k}$ is of the homotopy type of $\left.P^{k}\right)$ :

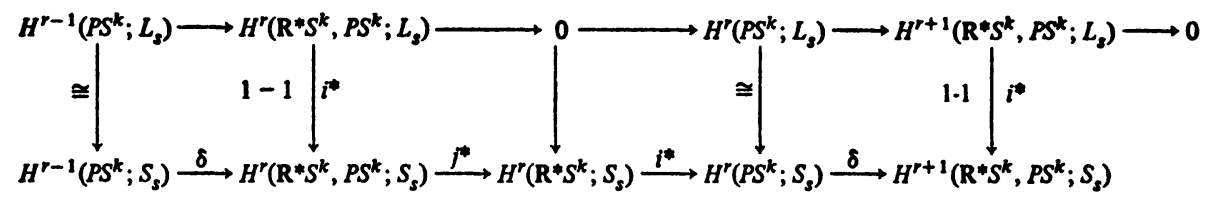

By a simple diagram-chasing argument, we are done.

Notation. If $y \in \operatorname{Ker} \delta^{r}$, we shall let $[y] \in \operatorname{Ker} \delta^{r} / \operatorname{Im} \delta^{r-1}$ be the class represented by $y$.

In the following lemmas, we shall assume that $n=2 k, k \geqslant 3$.

We examine a particular case. For any integer $s$, let $N_{s}^{n}$ be the total space of a real $(n-2)$-plane bundle $\xi_{x}^{n-2}$ over $P^{2}$ such that $\xi_{s}^{n-2} \oplus 3 h$ is stably equivalent to $s h$, the $s$-fold Whitney sum of the canonical line bundle, $h$. Clearly, $N_{s}^{n}=N_{s+4}^{n}$, since $h$ has order 4 in $K$-theory. If $\tau_{s}$ is the tangent bundle of $N_{s}^{n}=N, \tau_{s}$ is stably equivalent to $s h$, hence $w_{1} N=\left(\begin{array}{l}s \\ 1\end{array}\right) m$ and $w_{2} N=\left(\begin{array}{l}5 \\ 2\end{array}\right) m^{2}$, where $m \in H^{1}\left(N ; Z_{2}\right)$ is the generator of that group.

Let $a \in \pi_{1} N \cong T_{2}$ be the generator. Then $\tilde{a} \in \pi_{2} N \cong Z$ is a generator, and $\tilde{a}^{a}=-\tilde{a}$. Let $f: S^{k} \rightarrow N$ be an embedding. Recall that $\Phi$ is the fiber of $\theta_{N}$. We have by 5.3.2 that

$$
F^{-1} \pi_{n-1} \theta_{N} \cong \begin{cases}Z+Z & \text { if } s \text { is even, } \\ Z+Z^{T} & \text { if } s \text { is odd, }\end{cases}
$$

where the first generator is represented by $1 \in Z \pi_{1}=\pi_{n-1} \Phi$, the second by $a \in \pi_{n-1} \Phi ;$ and also that

$$
F^{-1} \pi_{n} \theta_{N} \cong \begin{cases}Z^{T}+Z_{2}+Z+Z_{2} & \text { if } s \text { is even, } \\ Z^{T}+Z_{2}+Z^{T}+Z_{2} & \text { if } s \text { is odd, }\end{cases}
$$

where the generators are represented, respectively, by $1 \otimes a, 1 \otimes \eta, a \otimes \tilde{a}$, $a \otimes \eta \in Z \pi_{1} \otimes\left(\pi_{2} \oplus H_{2}\right)=\pi_{n} \Phi$.

Let $B=\mathbf{R}^{*} S^{k}$. Since $f$ is an embedding, $F$ has a lifting to $Y_{N}$, thus $F^{-1} \theta_{N}$ has a section, i.e. $F^{-1} \theta_{N}$ is in the category $X_{B}^{+}$of $B$-sectioned fibrations. The following lemma is phrased in the notation of [10].

Lemma 6.1.2. If $N=N_{s}^{n}$, then the first two stages of the Postnikov tower for $F^{-1} \theta_{N}$ are given by $\left(B=\mathbf{R}^{*} S^{k}\right)$ :

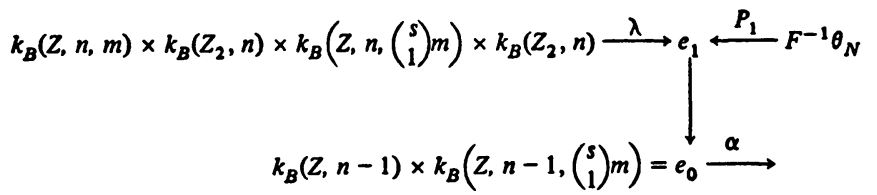

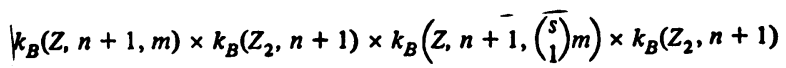


where $P_{1}$ induces isomorphism in homotopy through dimension $n$, and where

$$
\begin{aligned}
\alpha^{*}: \iota_{n+1} \otimes 1 \otimes 1 \otimes & \mapsto 0, \\
1 \otimes \iota_{n+1} \otimes 1 \otimes 1 & \mapsto\left(\mathrm{Sq}^{2}+m^{2}\right) \iota_{n-1} \otimes 1, \\
1 \otimes 1 \otimes \iota_{n+1} \otimes 1 & \mapsto 1 \otimes(\delta m) \iota_{n-1}, \\
1 \otimes 1 \otimes 1 \otimes \iota_{n+1} & \mapsto 1 \otimes\left(\mathrm{Sq}^{2}+\left(\begin{array}{c}
n-s \\
2
\end{array}\right) m^{2}\right) \iota_{n-1},
\end{aligned}
$$

where $\delta$ is the Bokstein homomorphism associated with the coefficient sequence $Z \rightarrow^{2} Z \rightarrow Z_{2}$.

We postpone the proof.

LEMMA 6.1.3. Let $a \in \pi_{1}, a^{2}=1$, and choose (jointly) $a \in \pi_{2}, 0<s=k_{a}$ $<4$ (cf. 5.2). Then in sequence (6.1-1):

(I) If $(-1)^{a}(-1)^{k}=-1, d_{2}[a]=a \otimes \tilde{a}+\left(\left(\frac{k-3}{2}\right)+\left(\begin{array}{c}n-s \\ 2\end{array}\right)\right) a \otimes \eta$;

(II) If $(-1)^{a}(-1)^{k}=1, \Phi_{2}[a] \in$ Coker $d_{2}$ is represented by $\left[a \otimes \tilde{a}+\left(\left({ }_{2}^{k-3}\right)\right.\right.$ $\left.\left.+\left(\begin{array}{c}n-s \\ 2\end{array}\right)+\left(\begin{array}{l}s \\ 1\end{array}\right)\right) a \otimes \eta\right]$.

Proof. We remind the reader that $(-1)^{a}=(-1)^{s}$, thus $\left(\begin{array}{l}s \\ 1\end{array}\right)=\left(\begin{array}{l}k \\ 1\end{array}\right) \bmod 2$ if $(-1)^{a}(-1)^{k}=1$.

Without loss of generality, $f$ is inessential, hence we may assume $f: S^{k} \rightarrow$ $N_{s}^{n} \subset M$. Since all constructions involved are natural with respect to inclusions of manifolds of the same dimension, we may assume that $M=N_{n}^{s}$. We have a commutative diagram with exact rows, where the first, second and fourth vertical arrows represent onto maps, by 6.1.1:

$$
\begin{array}{cccccccc}
H_{n-1}^{n-2} & \stackrel{d_{2}}{\rightarrow} & H_{n}^{n} & \stackrel{\lambda}{\rightarrow} & {\left[\mathrm{R}^{*} S^{k}, P S^{k} ; \theta_{M}\right]_{F}} & \stackrel{\rho}{\rightarrow} & H_{n-1}^{n-1} & \rightarrow 0 \\
\text { onto } j^{*} & & \begin{array}{c}
d_{2} \\
\text { onto } \downarrow j^{*}
\end{array} & & \downarrow j^{*} & & \text { onto } \downarrow j^{*} & \\
E_{2}^{-1, n-1} & \stackrel{d_{2}}{\rightarrow} & E_{2}^{0, n} & \stackrel{\lambda}{\rightarrow} & {\left[S^{k} \subset M\right]_{j}} & \stackrel{\rho}{\rightarrow} & E^{0, n-1} & \rightarrow 0
\end{array}
$$

It is sufficient to compute $d_{2}$ and $\Phi_{2}$ for the top row. Recall that $\cup m^{2}: H^{n-2}\left(\mathbf{R}^{*} S^{k}, P S^{k} ; Z_{2}\right) \rightarrow H^{n}\left(\mathbf{R}^{*} S^{k}, P S^{k} ; Z_{2}\right)$, while $\mathrm{Sq}^{2}$, on the same group, is nonzero if and only if $\left({ }^{k}{ }^{-3}\right)=1 \bmod 2$. Part (I) now follows immediately from 6.1.1 and 6.1.2, while (II) follows from 6.1.1, 6.1.2, and the results of [10].

6.2. Proof of 6.1.2. Let $p: \tilde{N} \rightarrow N$ be the universal covering of $N ; \tilde{N}=S^{2} \times$ $R^{n-2}$. Since $f$ may be assumed to be inessential, we may choose an embedding $\tilde{f}: S^{k} \rightarrow N$ such that $p \tilde{f}=f$. Note that $R^{\infty} \cong R^{\infty} \times R^{\infty}$. Thus we may choose an embedding $P$ such that the following diagram commutes, where $p_{1}$ is projection:

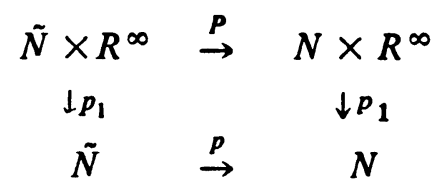


Let $\nabla N=\left(p^{2}\right)^{-1} \nabla N-\Delta \tilde{N} \subset R \tilde{N}$, and let $\nabla^{*} N \subset R^{*} \tilde{N}$ be the image of $\nabla N$. Let $\tilde{R} N=R \tilde{N}-\nabla N$ and $\tilde{R}^{*} N=R^{*} N-\nabla^{*} N$. Consider the diagram (where $p^{*}[x, y]=[p x, p y]$ for all $[x, y] \in \tilde{R}^{*} N$ ):

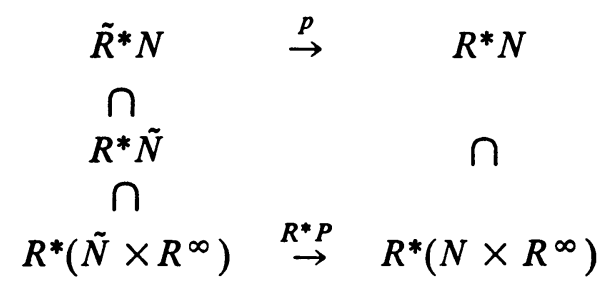

We have that $\Phi=$ fiber of $\tilde{R}^{*} N \subset R^{*}\left(\tilde{N} \times R^{\infty}\right)=$ fiber of $R^{*} N \subset R^{*}(N$ $\left.\times R^{\infty}\right)$.

LemMa 6.2.1 (HAEFLiger [4]). (I) As an algebra over $Z_{2}, H^{*}=H^{*}\left(R^{*}(\tilde{N}\right.$ $\left.\left.\times R^{\infty}\right) ; Z_{2}\right)=H^{*}\left(\Gamma S^{2} ; Z_{2}\right)$ is generated only by the elements: $m \in H^{1}, \Gamma \sigma \in$ $H^{2}$, and $\Sigma \sigma \in H^{4}$, subject only to the relations: $m \Gamma \sigma=(\Gamma \sigma)^{2}=\Gamma \sigma \Sigma \sigma=$ $(\Sigma \sigma)^{2}=0$.

(II) $\left(R^{*} i\right): H^{*} \rightarrow H^{*}\left(R^{*} N ; Z_{2}\right)$ is surjective, and its kernel is generated by $m^{n}$ and $m^{n-2} \Sigma \sigma$.

Lemma 6.2.2. The map $i^{*}: H^{*}\left(R^{*} \tilde{N} ; Z_{2}\right) \rightarrow H^{*}\left(\tilde{R}^{*} N ; Z_{2}\right)$ is injective, and its cokernel is generated over $Z_{2}$ by $\omega \in H^{n-1}\left(\tilde{R}^{*} N ; Z_{2}\right), m \omega$, and $m^{2} \mu$. Furthermore, $(\Gamma \sigma) \omega=m^{2} \omega$, and $\mathrm{Sq}^{i} \omega=\left(\begin{array}{c}n-s \\ i\end{array}\right) m^{i} \omega$ for all $i$.

Proof. We construct a map $\beta: R^{*} \tilde{N} \rightarrow \tilde{R}^{*} N$ such that $i \beta$ is homotopic to the identity on $R^{*} \tilde{N}$. Recall that $N=N_{s}^{n}$, the total space of $\xi_{s}^{n-2}=\xi$ over $P^{2}$. Choose a nonzero section $\chi^{*}$ of $\xi \otimes h$, and let $\chi: S^{2} \rightarrow S^{2} \times\left(R^{n-2}-\right.$ $\{0\}) \tilde{N}$ be the corresponding section of $\pi^{-1}(\xi \otimes h)$, the trivial $n$-bundle over $S^{2}$. Let $p_{1}: \tilde{N} \rightarrow S^{2}, p_{2}: \tilde{N} \rightarrow R^{n-2}$ be the projections. For all $0 \leqslant t \leqslant 1$, let $\beta_{t}: R^{*} \tilde{N} \rightarrow \tilde{R}^{*} N$ be defined as follows. If $[x, y] \in R^{*} \tilde{N}$, let $\rho=\rho(x, y)=$ $\left\|p_{1} x-p_{1} y\right\|$, and let:

$$
\beta_{t}[x, y]=\left\{\begin{array}{r}
{[x, y] \text { if } 1 \leqslant \rho \leqslant 2,} \\
{\left[\left(p_{1} x,(1+(\rho-1) t) p_{2} x+(1-\rho) t p_{2}\left(\chi\left(p_{1} x\right)\right)\right),\right.} \\
\left.p_{1} y,\left(1+(\rho-1) t p_{2} y+(1-\rho) t p_{2}\left(\chi\left(p_{1} y\right)\right)\right)\right] \\
\text { if } 0 \leqslant \rho \leqslant 1 .
\end{array}\right.
$$

Let $\beta=\beta_{1}$, which clearly has the desired property. Now the cofiber of the inclusion $\tilde{R}^{*} N \subset R^{*} \tilde{N}$ is the Thom complex of $\tau \otimes h$ over $N$, which we denote by $T C$, where $\tau$ is the tangent bundle of $N$. We have a commutative diagram with split exact columns, where each row is a long exact ThomGysin sequence, and all coefficients are $Z_{2}$ : 


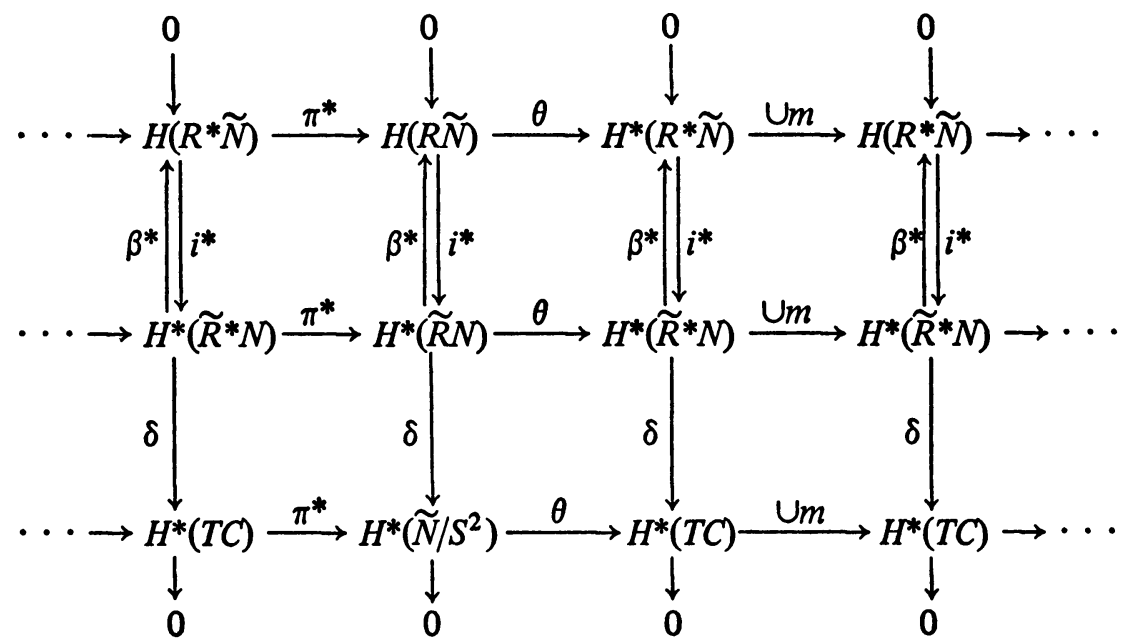

We now define $\omega \in H^{n-1}\left(\tilde{R}^{*} N ; Z_{2}\right)$ to be the unique element such that $\beta^{*} \omega=0$ and $\delta \omega=U$, the Thom class of $\tau \otimes h$. Now $H^{*}(T C)$ has only generators $U, m U$, and $m^{2} U$ over $Z_{2}$, and $\mathrm{Sq}^{i} U=\left(\begin{array}{c}n-s \\ i\end{array}\right) m^{i} U$ for all $i$, although $m^{3} U=0$. By exactness of the third row, $\theta\left(\sigma \cup \pi^{*} U\right)=\theta\left((\sigma \otimes 1) \cup \pi^{*} U\right)$ must be $m^{2} U$. Also, $\theta(\sigma \otimes 1)=\Gamma \sigma$, thus $(\Gamma \sigma) \omega=\theta\left((\sigma \otimes 1) \cup \pi^{*} \omega\right)=m^{2} \omega$. 6.2.2 then follows trivially.

Finally, Lemma 6.1.2 follows from routine Serre spectral sequence computation of the relative Postnikov tower of the inclusion $\tilde{R}^{*} N \subset R^{*}\left(\tilde{N} \times R^{\infty}\right)$.

\section{Affine groups.}

DefinItion 7.1. An affine group is a set $A$ together with an operation ${ }_{a}$ for each $a \in A$, such that $\left(A,{ }_{a}\right)$ is a group with identity $a$, and such that, for any $x, y, a, b \in A, x \cdot{ }_{a} y=x \cdot{ }_{b}(a)_{b}^{-1}{ }_{b} y$, where $(a)_{b}^{-1}$ is the inverse of $a$ under ${ }_{b}$. In addition, we say that $A$ is Abelian if ${ }_{a}{ }$ is commutative for all $a \in A$. (The operation shall then usually be denoted $+_{a}$.)

We remark that Becker's definition of affine group [1] is that of Abelian affine group.

An alternative definition, which is more natural to the constructions of this paper, is:

Definimion 7.2. An affine group is a set together with a ternary operation $\tau$ on $A$ such that (writing $\tau(x, y, z)=x y^{-1} z$ )

(i) $v w^{-1}\left(x y^{-1} z\right)=\left(v w^{-1} x\right) y^{-1} z$ (associative law),

(ii) $x y^{-1} y=y y^{-1} x=x$ (cancellation law).

In addition, we say that $A$ is $A$ belian if

(iii) $x y^{-1} z=z y^{-1} x$ (commutative law).

We leave the proof of the equivalence of 7.1 and 7.2 to the reader as an exercise (Hint: $x:_{a} y=x a^{-1} y$ ). Note that the empty set is an affine group. 
Letting $A$ be any affine group, we define two equivalence relations on $A \times A, R$ and $L$. For any $x, y, z, w \in A$, we say that $(x, y) R(z, w)$ if $y=x z^{-1} w$, and $(x, y) L(z, w)$ if $x=z w^{-1} y$. (Note that $R=L$ if $A$ is commutative.) Let $A^{R}=A \times A / R$, and $A^{L}=A \times A / L$. If $x, y \in A$, let $x^{-1} y \in A^{R}$ and $x y^{-1} \in A^{L}$ denote the elements represented by the ordered pair $(x, y)$. Both $A^{R}$ and $A^{L}$ are groups, under the operations $\left(x^{-1} y\right)\left(z^{-1} w\right)=$ $x^{-1}\left(y z^{-1} w\right)$, and $\left(x y^{-1}\right)\left(z w^{-1}\right)=\left(x y^{-1} z\right) w^{-1}$, respectively. We call $A^{R}$ and $A^{L}$ the right and left action groups of $A$, since $A^{R}$ acts on $A$ on the right, in the obvious manner suggested by the notation, and $A^{L}$ acts on $A$ on the left. We remark that $A^{R}$ and $A^{L}$ are isomorphic, canonically if $A$ is Abelian, in which case we write $A^{*}=A^{R}=A^{L}$, and $x-y$ instead of $x y^{-1}$ or $y^{-1} x$.

We remark that two affine groups are isomorphic if and only if their action groups are isomorphic. If $G$ is any group, $G$ may be taken to be an affine group by letting $x y^{-1} z$ have the usual meaning $(x-y+z$ if $G$ Abelian), in which case $G \cong G^{L}$ by letting $x$ correspond to $x 1^{-1}$.

If we say that an affine group $A$ is isomorphic to a group $G$, we shall mean that $A$ is isomorphic to $G$ considered as an affine group; equivalently, $A^{R} \cong G$ or $A^{L} \cong G$.

8. Correction. Recall diagram (2.6-1) in this paper, which is basically diagram (3.2-1) of [7]. We define a pair fibration ' $\zeta_{M}:\left({ }^{\prime} Y_{M},{ }^{\prime} Z_{M}\right) \rightarrow$ $\left({ }^{\infty} \mathbf{R} M,{ }^{\infty} P M\right)$ as follows: Let ' $Y_{M}$ be the space consisting of all paths $\sigma: I \rightarrow{ }^{\infty} \mathbf{R}^{*} M$ such that $\sigma(1) \in \mathbf{R} M$, and either $\sigma(0) \in{ }^{\infty} P M$ or $\sigma(1) \notin P M$. Let ' $Z_{M}$ be the space consisting of all paths $\sigma: I \rightarrow{ }^{\infty} P M$ such that the composition $I \rightarrow^{\circ}{ }^{\infty} P M \rightarrow M$ is constant. Let ' $\zeta_{M}:\left({ }^{\prime} Y_{M},{ }^{\prime} Z_{M}\right) \rightarrow$ $\left({ }^{\infty} \mathbf{R}^{*} M,{ }^{\infty} P M\right)$ be evaluation at 0 , a pair fibration, and let $\iota:$ ' $\zeta_{M} \subset \zeta_{M}$ be the obvious inclusion.

LEMMA 8.1. $\iota_{\#}:\left[\left(\mathbf{R}^{*} V, P V\right) ;{ }^{\prime} \zeta_{M}\right]_{F} \cong\left[\left(\mathbf{R}^{*} V, P V\right) ; \zeta_{M}\right]_{F}$.

PRoof. Let " $\zeta_{M}:\left(Y_{M}, Z_{M}\right) \rightarrow\left({ }^{\infty} \mathrm{R}^{*} M,{ }^{\infty} P M\right)$. Now the inclusion $i$ : (' $\left.Y_{M}, Z_{M}\right) \subset\left(Y_{M}, Z_{M}\right)$ is a fiber homotopy equivalence of pairs. Let $U$ $C^{\infty} \mathbf{R}^{*} M$ be a regular collar of ${ }^{\infty} P M$, such that $U \cap \mathbf{R} M$ is a regular collar of $P M$. Let $r_{t}:{ }^{\infty} \mathbf{R}^{*} M \rightarrow \mathbf{R}^{*} M, 0 \leqslant t \leqslant 1$, with $r_{0}=$ identity, be a strong deformation retraction of ${ }^{\infty} \mathbf{R}^{*} M$ onto the complement of $U$ such that $r_{t}\left(\mathbf{R}^{*} M\right) \subset \mathbf{R}^{*} M$ for all $t$. Let $\rho:{ }^{\infty} \mathbf{R}^{*} M \rightarrow I$ be continuous such that $\rho^{-1}\{0\}={ }^{\infty} P M$, and let $(j \sigma)(t)=r_{s}(t)$ for all $\sigma \in Y_{M}, t \in I$, where $s=$ $t \rho(\sigma(0))$. Clearly $j$ is the identity on ${ }^{\infty} P M$. The fact that $j$ is a pair fiberwise homotopy inverse of $i$ is trivial to verify. Thus $i_{\#}:\left[\left(\mathbf{R}^{*} V, P V\right) ; " \zeta_{M}\right]_{F} \simeq$ $\left[\left(\mathbf{R}^{*} V, P V\right) ; \zeta_{M}\right]_{F}$. Now $\pi_{k}\left({ }^{\prime} \zeta_{M}\right)=\pi_{k}\left(" \zeta_{M}\right)$ for all $k$, since ' $Z_{M} \rightarrow{ }^{\infty} P M$ and $Z_{M} \rightarrow{ }^{\infty} P M$ both have fibers of the homotopy type of $S^{n-1}$. By 2.4.1, we are done.

The error in the proof of Theorem 3.3.1 of [7] is the fact that if a lifting $\Phi$ 
of $F$ to $\zeta_{M}$ is given, the map $G[\Phi]$ in the diagram on p. 362 may not exist. If, on the other hand, $\Phi$ is a lifting of $F$ to ' $\zeta_{M}$ (which we may assume, by Lemma 8.1), existence of $G[\Phi]$ is assured. The remainder of the proof is valid. Theorem 3.3.2 of [7] can be corrected in a similar manner.

\section{BIBLIOGRAPHY}

1. J. C. Becker, Cohomology and the classification of liftings, Trans. Amer. Math. Soc. 133 (1968), 447-475. MR 38 \#5217.

2. Jean-Pierre Dax, Etude homotopique des espaces de plongements, Ann. Sci. École Norm. Sup. (4) 5 (1972), 303-377. MR 47 \#9643.

3. A. Haefliger, Plongements différentiables dans le domaine stable, Comment. Math. Helv. 37 (1962/63), 155-176. MR 28 \#625.

4. , Points multiples d'une application et produit cyclique réduit, Amer. J. Math. 83 (1961), 57-70. MR 22 \#11404.

5. I. M. Hall, The generalized Whitney sum, Quart. J. Math. Oxford Ser. (2) 16 (1965), 360-384. MR 32 \# 4698.

6. S.-T. Hu, Homotopy theory, Academic Press, New York and London, 1959. MR 21 \#5186.

7. L. L. Larmore, Obstructions to embedding and isotopy in the metastable range, Rocky Mountain J. Math. 3 (1973), 355-375. MR 50 \#8559.

8. , Twisted cohomology theories and the single obstruction to lifting, Pacific J. Math. 41 (1972), 755-769. MR 50 \# 5799.

9. L. L. Larmore and E. Thomas, Group extensions and principal fibrations, Math. Scand. 30 (1972), 227-248. MR 48 \# 7277.

10. Group extensions and twisted cohomology theories, Illinois J. Math. 17 (1973), 397-410. MR 49 \# 1517.

11. M. Mahowald and R. Rigdon, Obstruction theory with coefficients in a spectrum, Trans. Amer. Math. Soc. 204 (1975), 365-384.

12. Hans A. Salomonsen, On the existence and classification of differentiable embeddings in the metastable range (to appear).

Department of Mathematics, California State University, Dominiguez Hills, CaliFORNIA 90747 